\title{
Framing energetic top-quark pair production at the $\mathrm{LHC}$
}

\author{
Fabrizio Caola, ${ }^{a, b}$ Frédéric A. Dreyer, ${ }^{a}$ Ross W. McDonald ${ }^{a}$ and Gavin P. Salam ${ }^{a, c}$ \\ ${ }^{a}$ Rudolf Peierls Centre for Theoretical Physics, Clarendon Laboratory, \\ Parks Road, Oxford OX1 3PU, U.K. \\ ${ }^{b}$ Wadham College, \\ Oxford OX1 3PN, U.K. \\ ${ }^{c}$ All Souls College, \\ Oxford $O X 14 A L, U . K$. \\ E-mail: fabrizio.caola@physics.ox.ac.uk, \\ frederic.dreyer@physics.ox.ac.uk, ross.mcdonald@outlook.com, \\ gavin.salam@physics.ox.ac.uk
}

ABSTRACT: Top-quark pair production is central to many facets of LHC physics. At leading order, the top and anti-top are produced in a back-to-back topology, however this topology accounts only for a minority of the events with $\mathrm{TeV}$-scale momentum transfer that contain a $t \bar{t}$ pair. The remaining events instead involve the splitting of an initial or final-state gluon to $t \bar{t}$. We provide simple quantitative arguments that explain why this is the case, and examine the interplay between different topologies and a range of variables that characterise the event hardness. We then develop a method to classify the topologies of individual events and use it to illustrate our findings in the context of simulated events, using both top partons and suitably defined fiducial tops. For events with large $t \bar{t}$ invariant mass, we comment on additional features that have important experimental and theoretical implications.

KEYWORDS: QCD Phenomenology

ArXiv EPrint: 2101.06068 


\section{Contents}

1 Introduction 1

2 Theoretical considerations $\quad 3$

2.1 Event hardness variables and their leading-order behaviour 3

2.2 Topologies beyond LO 5

2.3 Interplay between topologies and hardness characterisation variable $\quad 9$

3 Parton-level (truth-top) analysis $\quad 10$

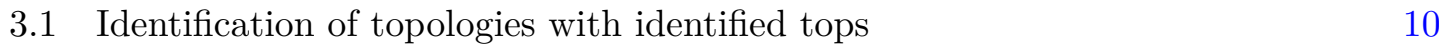

$\begin{array}{lll}3.2 & \text { Results } & 12\end{array}$

4 Particle-level (reconstructed top) analysis $\quad 16$

$\begin{array}{lll}4.1 & \text { Event analysis } & 16\end{array}$

$\begin{array}{lll}4.2 & \text { Top reconstruction } & 18\end{array}$

$\begin{array}{lll}4.3 & \text { Validation of reconstruction performance } & 18\end{array}$

$\begin{array}{lll}4.4 & \text { Results } & 20\end{array}$

5 Conclusions 23

A Leading-order distributions $\quad 24$

$\begin{array}{lll}\text { A.1 Distributions differential in } m_{t \bar{t}} & 24\end{array}$

A.2 Distributions differential in the top transverse momentum 26

B Comments on the $m_{t \bar{t}}$ distribution beyond LO 28

\section{Introduction}

Top quarks are among the most central objects to collider physics today. As the heaviest known fundamental particle, the top-quark has a unique place in the standard model (SM), the only particle with a Yukawa coupling close to unity, making it a key player in many Beyond Standard Model (BSM) scenarios and in discussions of the stability of the SM universe [1]. Top-quark pair production is an increasingly important input to fits of parton distribution functions (PDFs) [2-8]. It plays a crucial role in effective field theory (EFT) studies [9-13], provides an avenue to learning about the top-quark Higgs Yukawa coupling [14]. More generally top quarks are omnipresent in beyond standardmodel (BSM) searches, both as signal objects and for backgrounds (e.g. as a source of leptons and $b$ quarks). 
Early studies of top-quark production at Fermilab's Tevatron and CERN's Large Hadron Collider (LHC) were restricted to configurations where the top quarks had a transverse momentum that was comparable to the top-quark mass. Today, at each of the ATLAS and CMS collision points, the LHC has produced over a hundred thousand events with top quarks having a transverse momentum $p_{T}>500 \mathrm{GeV}$ and a couple of thousand events with $p_{T}>1 \mathrm{TeV}$ (all decay channels combined), with those numbers expected to increase by a factor of 25-30 at the high-luminosity LHC [15]. These large numbers of events provide an opportunity for percent-level precision across a wide kinematic range, with corresponding experimental measurements well underway [16-21]. This measurement programme will benefit a wide range of physics areas.

To reap these benefits, it will be necessary to achieve corresponding percent-level understanding of theoretical predictions and one may expect to leverage the impressive ongoing progress in perturbative calculations (for a review see ref. [22]). The reliability of this approach depends, however, on an assumption that the perturbative series is well behaved. A prerequisite is that the event topology that appears at LO, i.e. a $2 \rightarrow 2$ process with a back-to-back top-pair, dominates over topologies that start to arise only at higher orders, for example a boosted $t \bar{t}$ system recoiling against a hard jet. As we shall see, the extent to which this is true depends on the choice of observable used to characterise the event hardness. For some choices, the hierarchy holds as expected, but for other widely used choices of event hardness scale this is not the case, (e.g. for any observable that sums the transverse momenta of both jets and the top quarks). Accordingly, it is crucial to develop an understanding of the interplay between various widely used measures of the event hardness and the underlying topology of $t \bar{t}$ production. This is one of the main goals of this manuscript.

There are multiple potential benefits to having this understanding. For applications where precision is crucial, it can inform the choice of measurement observables. More generally, we will develop an approach for identifying the topology of any given event, so as to be able to separate out subsets of events that may probe different underlying physics in the specific application being considered, whether a PDF fit, an EFT fit or a BSM search.

This article is structured as follows. In section 2, we examine a range of variables used to characterise the hardness of $t \bar{t}$ events, examine the event topologies that can be produced at leading order (LO) and next-to-leading order (NLO), give a simple analysis of our expectations for their relative sizes, and finally consider the interplay between these topologies and the event hardness variables. In section 3 we introduce a procedure for identifying the topologies of individual events given the top quarks and a list of the other event particles, apply the procedure to simulated $t \bar{t}$ events, and compare the results to the expectations of section 2. In section 4 we combine this analysis with a fiducial reconstructed top-quark definition designed to identify top-quark candidates from the full set of finalstate particles across a wide range of transverse momenta. In section 5 , we conclude with a discussion of the implications of our results. Throughout this paper we will consider the semi-leptonic $t \bar{t} \rightarrow b \bar{b} \mu \nu_{\mu} j j$ decay channel, though the arguments that we make apply generally to any $t \bar{t}$ decay channel. 


\begin{tabular}{|cl|}
\hline Hardness variable & explanation \\
\hline$p_{T}^{\text {top,had }}$ & transverse momentum of hadronic top candidate \\
$p_{T}^{\text {top,lep }}$ & transverse momentum of leptonic top candidate \\
$p_{T}^{\text {top,max }}$ & $p_{T}$ of the top (anti-)quark with larger $m_{T}^{2}=p_{T}^{2}+m^{2}$ \\
$p_{T}^{\text {top,min }}$ & $p_{T}$ of the top (anti-)quark with smaller $m_{T}^{2}=p_{T}^{2}+m^{2}$ \\
$p_{T}^{\text {top,avg }}$ & $\frac{1}{2}\left(p_{T}^{\text {top,had }}+p_{T}^{\text {top,lep }}\right)$ \\
\hline$\frac{1}{2} H_{T}^{t \bar{t}}$ & with $H_{T}^{t \bar{t}}=m_{T}^{\text {top,had }}+m_{T}^{\text {top,lep }}$ \\
$\frac{1}{2} H_{T}^{t \bar{t}+\text { jets }}$ & with $H_{T}^{t \bar{t}+\text { jets }}=m_{T}^{\text {top,had }}+m_{T}^{\text {top,lep }}+\sum_{i} p_{T}^{j_{k, i}}$ \\
$m_{T}^{J, \text { avg }}$ & average $m_{T}$ of the two highest $m_{T}$ large- $R$ jets $\left(J_{1}, J_{2}\right)$ \\
\hline$\frac{1}{2} m^{t \bar{t}}$ & half invariant mass of $p^{t \bar{t}}=p^{\text {top,had }}+p^{\text {top,lep }}$ \\
\hline$p_{T}^{t \bar{t}}$ & transverse component of $p^{t \bar{t}}$ \\
$p_{T}^{j_{\psi \prime}}$ & transverse momentum of the leading small- $R$ non-top jet \\
\hline
\end{tabular}

Table 1. Variables that may be used to characterise a hard kinematic scale in events with a semileptonically decaying $t \bar{t}$ pair. All observables within a given group are identical to each other at leading order. The $j_{\ell, i}$ jets correspond to $R=0.4$ non-top jets, while the $J_{i}$ jets corresponds to large- $R$ jets (whose clustering inputs include the top quarks). Further details about the jet finding are given in sections 3 and 4 . The difference between $m_{T}$ and $p_{T}$ ordering for the $p_{T}^{\text {top,max }}$ and $p_{T}^{\text {top,min }}$ observables is largely immaterial.

\section{Theoretical considerations}

In this section we review various event-hardness measures, and discuss some basic expectations about their behaviour for events that involve large momentum-transfer and contain a $t \bar{t}$ pair.

\subsection{Event hardness variables and their leading-order behaviour}

We start by examining measures to characterise large momentum transfer in $t \bar{t}$ events, i.e. the event hardness, including a discussion of their leading-order distributions. A wide variety of such measures is used in the literature and we provide an illustrative selection of them in table 1, organised into groups that are identical at LO, i.e. for events that consist of just a single back-to-back $t \bar{t}$ pair.

The first set of observables simply measures the top-quark transverse momentum. They differ only in terms of which top-quark is used, which is why they are identical at LO $\left(\operatorname{order} \alpha_{s}^{2}\right.$ ). We also have an all-order relation between some of the observables, specifically ${ }^{1}$

$$
\frac{d \sigma}{d p_{T}^{\text {top,had }}}=\frac{d \sigma}{d p_{T}^{\text {top,lep }}}=\frac{1}{2}\left(\frac{d \sigma}{d p_{T}^{\text {top }, \text { max }}}+\frac{d \sigma}{d p_{T}^{\text {top,min }}}\right) .
$$

\footnotetext{
${ }^{1}$ The rightmost expression of our eq. (2.1) is referred to as $d \sigma / d p_{T, \text { avt }}$ in ref. [23]. This differs from our $d \sigma / d p_{T}^{\text {top,avg }}$ beyond LO.
} 
Note that we have chosen to define the "max/min" based on the value of $m_{T}$ rather than $p_{T}$, but results would be essentially unchanged if we instead used $p_{T}$. In the $\mathrm{LO}$ calculations that we report below, it will be convenient to have the shorthand $p_{T, t}$ for the transverse momentum of either of the top quarks.

The next set of observables provides measures of the hard scale of the event that at LO include the top-quark mass and transverse momentum. The $H_{T}^{t \bar{t}}$ variable is identical to the $H_{T}$ of Czakon, Heymes and Mitov [23] and of Catani et al. [24]. The $H_{T}^{t \bar{t}+\text { jets }}$ observable provides a democratic evaluation of the event hardness across all objects. At high scales it is very similar to the $m_{\text {eff }}$ variable used in supersymmetry searches, which is the scalar sum of the transverse momenta of all jets, leptons and missing momenta, see e.g. ref. [25]. ${ }^{2}$ The $m_{T}^{J \text {,avg }}$ quantity is based on large- $R$ jets, with the details of the jet finding discussed in more detail below. It is not part of the standard set of $t \bar{t}$ event-hardness measures, but we include it here because it gives a faithful reflection of the hardness of the main $2 \rightarrow 2$ scattering in the event, regardless of the precise role played by the top quarks in that scattering.

At leading order, in the limit where $p_{T, t}$ grows much larger than the top-quark mass $\left(m_{\text {top }}\right)$, all three observables in this group become identical to the $p_{T, t}$ type observables of the first group. Structurally, for $p_{T, t} \gg m_{\text {top }}$ but still significantly smaller than the collider centre-of-mass energy $\sqrt{s}$, the LO cross section is given by

$$
\frac{d \sigma}{d p_{T, t}^{2}}=\frac{\alpha_{s}^{2} \pi}{4 p_{T, t}^{4}}\left[c_{g g} \mathcal{L}_{g g}\left(4 p_{T, t}^{2} / s\right)+c_{q \bar{q}} \mathcal{L}_{q \bar{q}}\left(4 p_{T, t}^{2} / s\right)\right]
$$

Here the $c_{g g}$ and $c_{q \bar{q}}$ are numerical constants, of the order of 0.1 , which depend on how steeply the PDFs fall with increasing $x$. They are discussed in appendix A.2, together with our specific definition the partonic luminosities $\mathcal{L}_{g g}$ and $\mathcal{L}_{q \bar{q}}$. Since this is a LO calculation, for simplicity we have left out explicit renormalisation and factorisation scale dependence.

The next observable in table 1 is the invariant mass of the $t \bar{t}$ system. For $m_{t \bar{t}} \gg m_{\mathrm{top}}$, the $\mathrm{LO}$ distribution is

$$
\frac{d \sigma}{d m_{t \bar{t}}^{2}}=\frac{\alpha_{s}^{2} \pi}{m_{t \bar{t}}^{4}}\left[\left(\frac{1}{3} \ln \frac{m_{t \bar{t}}^{2}}{m_{\text {top }}^{2}}-\frac{7}{12}\right) \mathcal{L}_{g g}\left(m_{t \bar{t}}^{2} / s\right)+\frac{8}{27} \mathcal{L}_{q \bar{q}}\left(m_{t \bar{t}}^{2} / s\right)\right] .
$$

Relative to the result for the $p_{T, t}$ distribution, eq. (2.2), a key structural difference here is the presence of a factor $\ln \frac{m_{t \bar{t}}^{2}}{m_{\text {top }}^{2}}$ multiplying the gluon-gluon luminosity. One can understand the origin of this logarithm by considering fixed $m_{t \bar{t}}$ and examining the distribution of $\Delta y_{t \bar{t}}$, the difference in rapidity between the top and anti-top. At large $\Delta y_{t \bar{t}}$, the gluon-fusion contribution is dominated by a $t$-channel top-quark exchange diagram and the cross section becomes a constant, independent of $\Delta y_{t \bar{t}}$. Integrating over $\Delta y_{t \bar{t}}$ up to its kinematic limit,

$$
\Delta y_{t \bar{t}}^{\max }=\cosh ^{-1}\left(\frac{m_{t \bar{t}}^{2}}{2 m_{\mathrm{top}}^{2}}-1\right)=\ln \frac{m_{t \bar{t}}^{2}}{m_{\mathrm{top}}^{2}}+\mathcal{O}\left(\frac{m_{\mathrm{top}}^{2}}{m_{t \bar{t}}^{2}}\right),
$$

\footnotetext{
${ }^{2}$ The reason for not directly including $m_{\text {eff }}$ in this paper is that it cannot be meaningfully used with top partons, but only with top decay products.
} 
then yields the logarithmic factor seen in eq. (2.3), cf. appendix A.1. The large- $\Delta y_{t \bar{t}}$ enhancement of the gluon-fusion versus $q \bar{q}$ contributions provides a potentially powerful handle for separately constraining the $q \bar{q}$ and $g g$ luminosities in PDF fits.

Note that at the large $\Delta y_{t \bar{t}}$ values that dominate the gluon-fusion contribution to eq. (2.3), the top-quark transverse momentum $p_{T, t}$ is much smaller than its $\Delta y_{t \bar{t}}=0$ value of approximately $m_{t \bar{t}} / 2$. This is the reason why one should be wary of using $m_{t \bar{t}}$ as a renormalisation and factorisation scale for calculating the $m_{t \bar{t}}$ distribution at large $m_{t \bar{t}}$ values, and why one would expect the use of $\mu=m_{t \bar{t}}$ to lead to poor stability, as observed in ref. [23]. ${ }^{3}$ In practice, once one uses a dynamical scale, the LO result is no longer flat in $\Delta y_{t \bar{t}}$, but is sensitive to the varying scale of both $\alpha_{s}$ and the PDF, and typically results in a $\Delta y_{t \bar{t}}$ distribution that is peaked at large $\Delta y_{t \bar{t}}{ }^{4}$. Some plots illustrating these points are given at LO in appendix A and at NLO in section 3.2 and appendix B.

The importance of large $\Delta y_{t \bar{t}}$ values makes the $m_{t \bar{t}}$ observable subtle both theoretically and experimentally. The theoretical subtleties are discussed briefly in appendix B and have two main facets: firstly, for $t$-channel top-quark exchange a rich structure of logarithmically enhanced terms, $\alpha_{s}^{n} \ln ^{m} m_{t \bar{t}} / m_{\text {top }}$, develops beyond LO. Secondly, contributions from 4-top final states with $t$-channel gluon exchange, and $\mathrm{EW} b \bar{b} \rightarrow t \bar{t}$ diagrams with $t$-channel $W$ exchange both scale as $1 /\left(m_{\text {top }}^{2} m_{t \bar{t}}^{2}\right)$ rather than $1 /\left(m_{t \bar{t}}^{4}\right)$. For sufficiently large $m_{t \bar{t}}$ values they dominate over other contributions. At the LHC, they bring only a small contribution, because of suppression by phase-space and couplings, but this would no longer be the case at a $100 \mathrm{TeV} p p$ collider.

Experimentally there are also at least two facets to the subtleties for the $m_{t \bar{t}}$ observable. Firstly, large $\Delta y_{t \bar{t}}$ implies large rapidities for at least one of the two tops, which may then be beyond the detector acceptance, notably for $b$-tagging and lepton identification. Secondly, the spread of $p_{T, t}$ values across the range of $\Delta y_{t \bar{t}}$ adds the complication that standard measurement approaches, which use either resolved or boosted top reconstruction techniques, cannot reconstruct tops across the whole range of $\Delta y_{t \bar{t}}$. We will return to this issue below.

The last two observables that we show in table $1, p_{T}^{t \bar{t}}$ and $p_{T}^{j_{\psi 1}, 1}$, are identically zero at order $\alpha_{s}^{2}$. In the absence of $p_{T}$ and rapidity acceptance cuts for jets, the two observables are identical at order $\alpha_{s}^{3}$, as long as one has a concrete scheme that separates the top decay products from other event particles. Given that they start at one order in $\alpha_{s}$ higher than the other observables, one might expect them to have significantly smaller cross sections (in particular as compared to the $p_{T}$ type observables). As we shall see, this is not the case.

\subsection{Topologies beyond LO}

Having reviewed the key characteristics of the LO distributions, we can now turn to the main topic of this paper, the question of topologies beyond LO and the interplay between topologies and event hardness scales.

\footnotetext{
${ }^{3}$ Similar considerations apply to the measurement of the running top-quark mass as a function of $m_{t \bar{t}}[26]$. Indeed, at high $m_{t \bar{t}}$ the sensitivity of the cross section to the top-quark mass will come predominantly from the region close to the kinematic limit of $\Delta y_{t \bar{t}}$, where the largest virtuality of the produced or exchanged top quarks is much closer to $m_{\text {top }}$ than to $m_{t \bar{t}}$.

${ }^{4}$ This feature was observed numerically in the context of FCC-hh studies in section 12.3 of ref. [27].
} 
flavour creation

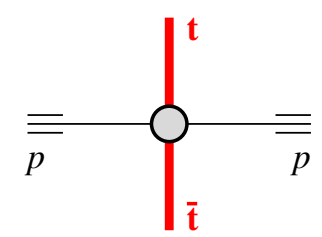

flavour excitation

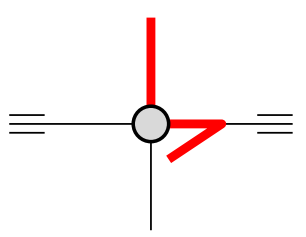

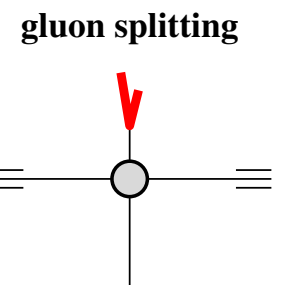

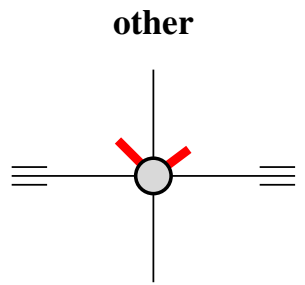

Figure 1. Illustration of classes of event topology for top production. Thick red lines represent top (anti-)quarks, while thin black lines represent light partons (quarks or gluons). Protons are depicted as entering from the left and right-hand sides.

The topologies that we will consider are illustrated in figure 1 and will be familiar to some readers from older discussions of $b$-quark production. The flavour creation (FCR) configuration is the dominant mechanism for top production at low transverse momentum. It is the only topology that is present at leading order (LO) in a strong-coupling perturbative expansion. In flavour excitation (FEX), a $t \bar{t}$ pair can be produced by an initial state splitting, with one of the pair undergoing a large momentum-transfer scattering with a light parton. Gluon splitting (GSP) involves production of a $t \bar{t}$ pair during jet fragmentation. Both FEX and GSP start at next-to-leading order (NLO). Finally some events do not readily fall into any of these categories, for example two high-transverse momentum light-flavour jets plus a (relatively) soft additional gluon that splits to $t \bar{t}$. These arise only at NNLO and beyond.

Relative to LO, the FEX and GSP topologies involve a factor $\alpha_{s} \ln p_{T} / m_{\text {top }}$, where $p_{T}$ is generally the transverse momentum of the hardest object in the event. The $\ln p_{T} / m_{\text {top }}$ factor that arises at the LHC is typically not large: e.g. for $p_{T} \sim 1 \mathrm{TeV}$, it is of the order 2, which would not be expected to compensate for the extra power of $\alpha_{s}$ relative to LO, and one might expect FEX and GSP to be small compared to FCR. ${ }^{5}$ As we shall see, this intuition misses important considerations. To help understand this, table 2 shows the different factors that come into the calculation of the cross section for the FCR, FEX and GSP topologies. We consider a $2 \rightarrow 2$ hard scattering energy of $2 \mathrm{TeV}$ and take the case of 90 degree scattering in the centre of mass, which dominates high- $p_{T}$ production. This corresponds to each outgoing object from the $2 \rightarrow 2$ scattering having a transverse momentum of $1 \mathrm{TeV}$ and identical rapidity.

The first point that we highlight is that the underlying $2 \rightarrow 2$ matrix elements for the FCR process are an order of magnitude smaller than for FEX and GSP. To illustrate the origin of this analytically in one simple case, consider $90^{\circ}$ scattering in the limit $p_{T} \gg m_{\text {top }}$, and compare for example the squared matrix element relevant for the $q_{i} \bar{q}_{i} \rightarrow t \bar{t}$ channel of FCR (cf. [34] or [35]),

$$
\frac{1}{g^{4}} \sum_{\text {spin,colour }}\left|\mathcal{M}_{q \bar{q} \rightarrow q^{\prime} \bar{q}^{\prime}}\right|^{2}=\frac{C_{F}}{N_{C}} \frac{\hat{t}^{2}+\hat{u}^{2}}{\hat{s}^{2}}=\frac{C_{F}}{N_{C}} \cdot \frac{1}{2},
$$

\footnotetext{
${ }^{5}$ At a $100 \mathrm{TeV} p p$ collider, the logarithms can be larger, which might then at first sight explain the observation in section 12.3 of ref. [27] that GSP contributes significantly to high- $p_{T}$ top production.
} 


\begin{tabular}{|cccccc|}
\hline topology & channel & $|\mathrm{ME}|^{2}$ & luminosity & FS splitting & product \\
\hline \multirow{2}{*}{ FCR } & $g g \rightarrow t \bar{t}$ & 0.15 & 0.16 & 1 & 0.024 \\
& $q_{i} \bar{q}_{i} \rightarrow t \bar{t}$ & 0.22 & 0.13 & 1 & 0.028 \\
\hline \multirow{2}{*}{ FEX } & $t g \rightarrow t g$ & 6.11 & 0.0039 & 1 & 0.024 \\
& $t \Sigma \rightarrow t \Sigma$ & 2.22 & 0.0170 & 1 & 0.038 \\
\hline \multirow{2}{*}{ GSP } & $g g \rightarrow g g(\rightarrow t \bar{t})$ & 30.4 & 0.16 & $\mathcal{P}_{g \rightarrow t \bar{t}} \simeq 0.004$ & 0.020 \\
& $g \Sigma \rightarrow g(\rightarrow t \bar{t}) \Sigma$ & 6.11 & 1.22 & $\mathcal{P}_{g \rightarrow t \bar{t}} \simeq 0.004$ & 0.031 \\
& $q \bar{q} \rightarrow g g(\rightarrow t \bar{t})$ & 1.04 & 0.13 & $\mathcal{P}_{g \rightarrow t \bar{t}} \simeq 0.004$ & 0.001 \\
\hline
\end{tabular}

Table 2. Factors contributing to the top-production cross section for a variety of partonic scattering channels. In each case the $2 \rightarrow 2$ squared matrix element $\left(|\mathrm{ME}|^{2}\right.$, with a $g^{4}=\left(4 \pi \alpha_{s}\right)^{2}$ factor stripped off as in eqs. $(2.5),(2.6)$ ) is given in the massless limit (valid when $p_{T} \gg m_{t}$ ), for $90^{\circ}$ scattering in the partonic centre-of-mass frame. The partonic luminosities, defined as in eq. (A.2), are given for a proton-proton centre of mass energy of $\sqrt{s}=13 \mathrm{TeV}$ and for producing a partonic system mass of $\sqrt{\hat{s}}=2 \mathrm{TeV}$. We set the factorisation scale to $\mu=1 \mathrm{TeV}$. $\Sigma$ denotes a sum over all (non-top) quark and anti-quark flavours. The luminosities have been evaluated with the PDF4LHC15_nnlo_mc [28] set, re-evolved in a six-flavour scheme with HOPPET [29] using NNLO splitting and threshold-matching functions [30-33]. The final-state splitting probability $P_{g \rightarrow t \bar{t}}$ is obtained using eq. (2.9). The results in the final column are to be taken as order of magnitude estimates, illustrating the commensurate sizes of different channels.

to that involved in the $q t \rightarrow q t$ channel of FEX,

$$
\frac{1}{g^{4}} \sum_{\text {spin, colour }}\left|\mathcal{M}_{q q^{\prime} \rightarrow q q^{\prime}}\right|^{2}=\frac{C_{F}}{N_{C}} \frac{\hat{s}^{2}+\hat{u}^{2}}{\hat{t}^{2}}=\frac{C_{F}}{N_{C}} \cdot 5 .
$$

The Mandelstam invariants are $\hat{s}=4 p_{T}^{2}$ and $\hat{t}=\hat{u}=-2 p_{T}^{2}$, and as a result the FEX channel has a squared matrix element that is ten times larger than the FCR channel.

A second factor that is relevant is the partonic luminosity. For the FEX channels, the incoming top is produced by an initial-state $g \rightarrow t \bar{t}$ splitting, so ultimately the cross section is driven by $g g$ and $g \Sigma$ luminosities, where $\Sigma$ is the sum of all light (anti-)flavours. The top-quark luminosity then involves a factor $\alpha_{s} \ln p_{T} / m_{\text {top }}$, which gives a smaller luminosity than either the $g g$ or $q_{i} \bar{q}_{i}$ luminosities that were relevant for the FCR case. Ultimately the larger matrix element compensates for the reduced luminosities and the FEX process has a cross section that is comparable to that for FCR.

A similar set of features emerges also for the GSP case. Here the $\alpha_{s} \ln p_{T} / m_{\text {top }}$ factor appears for the final-state splitting rather than an initial state one. It is straightforward to use massive splitting functions [36] to evaluate the leading-order probability $\mathcal{P}_{g \rightarrow t \bar{t}}$ for $g \rightarrow t \bar{t}$ splitting with the $t \bar{t}$ pair separated by distance $\Delta R_{t \bar{t}}<R$, where $\Delta R_{t \bar{t}}^{2}=\left(y_{t}-\right.$ $\left.y_{\bar{t}}\right)^{2}+\left(\phi_{t}-\phi_{\bar{t}}\right)^{2}$ and $y_{t}$ and $\phi_{t}$ are respectively the rapidity and azimuth of the top. For a gluon transverse momentum of $p_{T}$, and with the conditions $p_{T} R \gg m_{\mathrm{top}}$ and $R \ll 1$, the result is

$$
\mathcal{P}_{g \rightarrow t \bar{t}}=\frac{\alpha_{s} T_{R}}{2 \pi} \frac{2}{3}\left(\ln \frac{p_{T, t}^{2} R^{2}}{m_{\mathrm{top}}^{2}}-\frac{23}{6}\right) .
$$


In practice, the regime of $p_{T}=1 \mathrm{TeV}$ is not sufficiently asymptotic for this expression to hold, as one can see by substituting $R=1$ and observing that the result is negative. To obtain a more reliable estimate, we maintain the conditions $p_{T} \gg m_{\text {top }}$ and $R \ll 1$, but relax the constraint on $p_{T} R / m_{\text {top }}$. The resulting expression is a little cumbersome, ${ }^{6}$ but the following parametrisation reproduces the correct result to better than $1 \%$ for all values of $p_{T} R / m_{\text {top }}$

$$
\mathcal{P}_{g \rightarrow t \bar{t}} \simeq \frac{\alpha_{s} T_{R}}{2 \pi} \frac{1}{3} \frac{\ln \left(1+e^{4 x-23 / 3}+e^{2 x} / 10\right)}{1-0.101 e^{-(x-2.2)^{2} / 2.3}}, \quad x=\ln \frac{p_{T, t} R}{m_{\text {top }}}
$$

For finite values of $R$, we expect additional corrections that most likely would scale as $R^{2}$, as found for example in a small- $R$ jet context [37] (there the coefficient of $R^{2}$ was small). At this stage, there is some freedom in the $R$ value that we choose in order to define the gluon-splitting. Insofar as we are interested mainly in an order-of-magnitude estimate of $g \rightarrow t \bar{t}$, we evaluate eq. (2.9) with $R=1$, ignoring potential $R^{2}$-suppressed corrections. Substituting $p_{T, t}=1 \mathrm{TeV}$ and $m_{\mathrm{top}}=173 \mathrm{GeV}$ and $\alpha_{s}(1 \mathrm{TeV})=0.089$, this yields the result for $\mathcal{P}_{g \rightarrow t \bar{t}}$ shown in table 2. We see that, like FEX, the GSP topology is also comparable to the FCR one.

Were we to consider significantly harder events (e.g. at a $100 \mathrm{TeV}$ collider) or b-quarks instead of top quarks, the logarithmic factors would start to become large, further enhancing the FEX and GSP contributions relative to FCR. This is consistent with earlier findings of large relative FEX and GSP contributions to high- $p_{T} b$-jet production [38].

The analysis shown in table 2 is not intended to give precise predictions for the relative sizes of different topologies. Nevertheless it shows that, despite their being suppressed by a power of $\alpha_{s}$, the (NLO) FEX and GSP topologies are numerically comparable to the LO FCR topology at the TeV scale. ${ }^{7}$ By framing the discussion in terms of an asymptotic limit where $p_{T} \gg m_{\text {top }}$, we avoided providing a rigorous definition of the FCR, FEX and GSP topologies. If one wishes to study actual events, whether in fixed-order QCD, or at particle-level in experiments, a precise definition becomes necessary. This will be the topic of section 3 .

\footnotetext{
${ }^{6}$ Starting from the massive splitting function, one introduces $\mu=m_{\mathrm{top}} /\left(p_{T, t} \Delta R\right.$ ) (where $\Delta R$ is the separation between the top and anti-top), performs the logarithmic integral over $\mu$, and after rewriting the expression in terms $y$, which is the solution of $\mu^{2}=-y^{2} /(1-y)^{4}$, integrates over $z$. One obtains

$$
\begin{aligned}
\mathcal{P}_{g \rightarrow t \bar{t}}= & \frac{\alpha_{s} T_{R}}{2 \pi}\left(-\frac{4\left(y^{4}-9 y^{3}+23 y^{2}-9 y+1\right)}{3(y-1)^{3} \sqrt{y^{2}-6 y+1}} \tanh ^{-1}\left(\frac{1-y}{\sqrt{y^{2}-6 y+1}}\right)+\right. \\
& \left.+\frac{2\left(y^{4}+y^{3}+3 y^{2}+y+1\right) \log (y)}{3(y-1)^{3}(y+1)}-\frac{23}{9}\right)
\end{aligned}
$$

and the solution to take for $y$ is $y=1-\frac{i}{2 \mu}+\frac{(1-i) \sqrt{4 \mu-i}}{2 \sqrt{2} \mu}$.

${ }^{7}$ This finding is reminiscent of the observation of giant $K$-factors discussed for example in ref. [39] for vector-boson plus jet production, though the $K$-factors in the $t \bar{t}$ case are less extreme.
} 


\begin{tabular}{|cccc|}
\hline Hardness variable & FCR & FEX & GSP \\
\hline$p_{T}^{\text {top,had }}$ & $\checkmark$ & $\checkmark$ & \\
$p_{T}^{\text {top,lep }}$ & $\checkmark$ & $\checkmark$ & \\
$p_{T}^{\text {top,max }}$ & $\checkmark$ & $\checkmark$ & \\
$p_{T}^{\text {top,min }}$ & $\checkmark$ & & \\
$p_{T}^{\text {top,avg }}$ & $\checkmark$ & & \\
\hline$\frac{1}{2} H_{T}^{t \bar{t}}$ & $\checkmark$ & & \\
$\frac{1}{2} H_{T}^{t \bar{t} \text { jets }}$ & $\checkmark$ & $\checkmark$ & $\checkmark$ \\
$m_{T}^{J \text {,avg }}$ & $\checkmark$ & $\checkmark$ & $\checkmark$ \\
\hline$\frac{1}{2} m^{t \bar{t}}$ & $\checkmark$ & & \\
\hline$p_{T}^{t \bar{t}}$ & & $\checkmark$ & $\checkmark$ \\
$p_{T}^{j_{k, 1}}$ & & $\checkmark$ & $\checkmark$ \\
\hline
\end{tabular}

Table 3. Summary of the topologies expected to contribute dominantly to the distributions of different hardness variables, when these are large relative to $m_{\text {top }}$. See text for details and table 1 for definitions of the hardness variables.

\subsection{Interplay between topologies and hardness characterisation variable}

Before turning to detailed topology definitions, we discuss the interplay between the topologies of figure 1 and the event hardness variables of table 1 . While, at the $\mathrm{TeV}$ scale, all three main topologies have comparable cross sections for comparable hardness of the underlying $2 \rightarrow 2$ scattering, their relative contributions to the differential distribution of some specific event hardness variable depends significantly on the choice of variable. A key principle to remember in the discussion is that each of the topologies has a cross section that falls steeply as a function of the underlying $2 \rightarrow 2$ transverse momentum $p_{T}^{2 \rightarrow 2}$, say as $\sim 1 /\left(p_{T}^{2 \rightarrow 2}\right)^{k}$ with some positive power $k$. Consider a specific value $V$ of a given hardness variable. If $p_{T}^{2 \rightarrow 2}$ is significantly larger than $V$ in some topology, its contribution to the bin around $V$ will be suppressed relative to another topology for which $p_{T}^{2 \rightarrow 2}$ is comparable to $V$. Equivalently, a topology where $V$ ends up being significantly smaller than $p_{T}^{2 \rightarrow 2}$ will be suppressed relative to a topology where $V$ is similar to $p_{T}^{2 \rightarrow 2}$. On this basis we can work out which topologies will be relevant for which hardness variable, and the conclusions are summarised in table 3 .

Specifically, we see that the first group of hardness variables in tables 1 and 3 , the $p_{T}^{\text {top }}$ set of variables, splits into two sub-groups. The first three variables $p_{T}^{\text {top,had }}, p_{T}^{\text {top,lep }}$, $p_{T}^{\text {top,max }}$ share the characteristic that they can be commensurate with $p_{T}^{2 \rightarrow 2}$ if at least one of the two tops is hard. Therefore we expect the distributions of these variables to receive significant contributions from the FCR and FEX topologies, ${ }^{8}$ but not from GSP (because

\footnotetext{
${ }^{8}$ In the asymptotically dominant limit where the softer of the two tops has negligible $p_{T}$ compared to the harder one, the FEX contribution for $p_{T}^{\text {top,had }}$ and $p_{T}^{\text {top,lep }}$ is half that for $p_{T}^{\text {top,max }}$. This will, however, not
} 
neither of the tops carries the full $p_{T}$ of the underlying hard process). In contrast, for $p_{T}^{\text {top,min }}$ and $p_{T}^{\text {top,avg }}$ to be commensurate with $p_{T}^{2 \rightarrow 2}$, both tops need to be hard, and so we expect significant contributions mainly from FCR.

The next set of variables in tables 1 and 3 also splits into two groups. The $\frac{1}{2} H_{T}^{t \bar{t}}$ variable is commensurate with $p_{T}^{2 \rightarrow 2}$ only if both tops are hard, i.e. we expect contributions mainly from FCR. In contrast, $\frac{1}{2} H_{T}^{t \bar{t}+\text { jets }}$ and $m_{T}^{J, \text { avg }}$ are always commensurate with $p_{T}^{2 \rightarrow 2}$, regardless of the underlying topology, and so we expect contributions from FCR, FEX and GSP. ${ }^{9}$

The $\frac{1}{2} m^{t \bar{t}}$ variable is special, as discussed in section 2.1 and appendix B. We do not expect significant FEX or GSP contributions associated with NLO matrix elements that are larger than the LO one, and on that basis would expect it to be dominated by FCR. However if $\log m^{t \bar{t}} / m_{\text {top }}$ becomes large enough, the discussion of appendix B implies that the largest log-enhanced contributions (e.g. $\alpha_{s}^{3} \ln ^{3} m^{t \bar{t}} / m_{\text {top }}$ terms) would include FEX-like topologies. At LHC energies, logarithms are not yet quite large enough to override the main perturbative hierarchy and accordingly we remain with the expectation that the $m^{t \bar{t}}$ distribution should mainly involve the FCR topology.

The last two variables that we consider are $p_{T}^{t \bar{t}}$ and $p_{T}^{j_{\psi, 1}}$, which are identical at NLO. They are commensurate with $p_{T}^{2 \rightarrow 2}$ only for the topologies with a hard non-top jet, i.e. for the FEX and GSP topologies.

The issue of the relevant topologies is not the only aspect that contributes to the size of the final cross sections for different hardness variables. We will comment on other aspects as they arise in the sections below.

\section{Parton-level (truth-top) analysis}

If we are to explore the relevance of different topologies in actual (simulated or experimentally observed) $t \bar{t}$ events, it becomes necessary to develop techniques to identify the $t \bar{t}$ event topologies based on the momenta of the top quarks and the other event particles. Such techniques need to be applicable even outside the asymptotically high-scale limit that formed the conceptual basis of the discussion in section 2 .

As a first step, we imagine a situation where we have full kinematic information about the top and anti-top quarks and that we can separate out all particles that do not stem from the $t \bar{t}$ decays. In section 3.1 we outline a simple algorithm to assign a classification of the topology for any given event. Then in section 3.2 we apply the algorithm to simulated parton-level events and compare the results to the expectations from section 2 .

\subsection{Identification of topologies with identified tops}

We consider a procedure for events with exactly one $t \bar{t}$ pair, and follow a two-stage reconstruction procedure, set out as Algorithm 1.

\footnotetext{
be unambiguously visible later when we compare FEX to FCR: the LO property that the FCR distributions of $p_{T}^{\text {top,had }}, p_{T}^{\text {top,lep }}$ and $p_{T}^{\text {top,max }}$ are identical is broken from NLO onwards, with the $p_{T}^{\text {top,max }}$ distribution being larger than the others.

${ }^{9}$ This is qualitatively similar to observations about $H_{T}$-type observables made some time ago in the context of $Z+$ jet studies [39].
} 


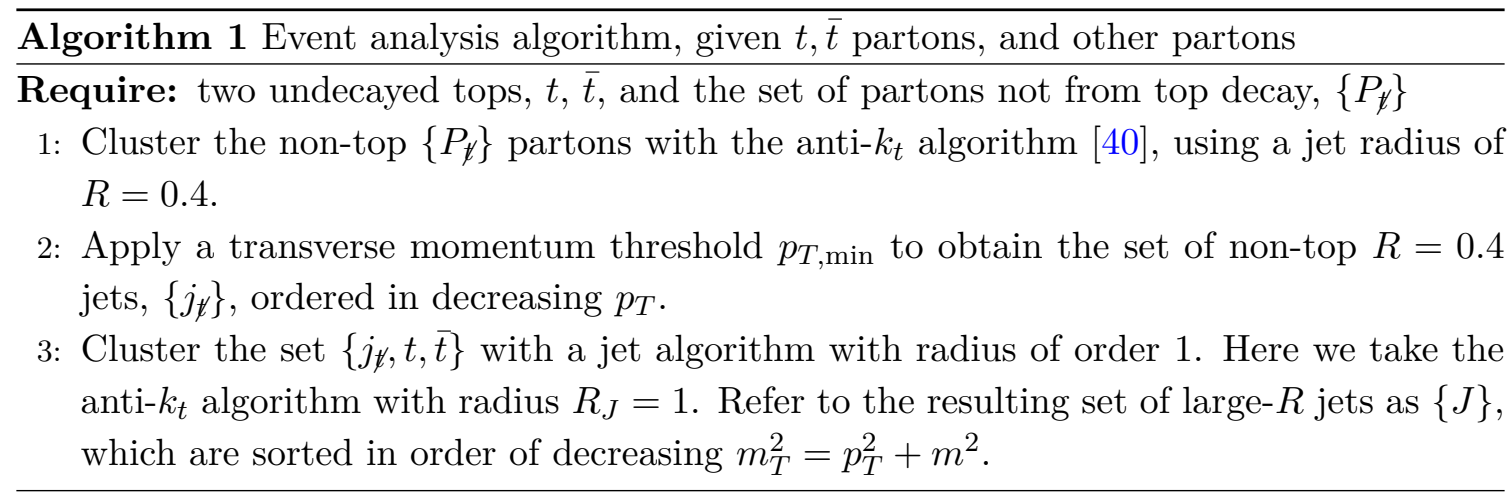

In the first stage we obtain $R=0.4$ jets from all objects other than the top quarks. This is the output of step 2 of the algorithm. The $R=0.4$ jet radius ensures that lowmomentum particles from the underlying event (UE) and pileup do not too significantly affect the momenta of genuinely hard jets. The $p_{T, \min }$ cut on the jets ensures that jets composed primarily of low-momentum particles from UE and pileup do not significantly affect variables that sum over multiple jets, such as $H_{T}^{t \bar{t}+\text { jets }}$. In this section we imagine a perfect detector, and apply no rapidity acceptance cuts, neither on the top quarks nor on the jets.

Step 3 of the algorithm then clusters the top quarks and the $R=0.4$ jets together using a jet radius of 1 . When there are jets or top quarks at high transverse momentum, this step effectively treats them democratically, reflecting a view that the top quarks are akin to light partons, and should be included in the clustering on the same footing as other partons. Taking $R \simeq 1$ is the natural choice for separating initial-state and final-state perturbative-QCD radiation [41]. The use of $R=0.4$ jets (with a $p_{T, \text { min }}$ threshold) as the input to the large- $R$ clustering ensures that the large- $R$ jets are kept relatively free of underlying-event and pileup contamination, in the same way as for observables such as $H_{T}^{t \bar{t}+\text { jets }}$ that sum over multiple jets. This clustering of smaller-radius jets into a larger radius system is reminiscent of CMS's radiation recovery procedure in dijet resonance searches [42] and bears similarities also to the use of filtering [43] or trimming [44] with large- $R$ jets for resonance reconstruction in ref. [45].

Sorting the large- $R$ jets $\{J\}$ in order of decreasing $m_{T}^{2}$ ensures that for low- $p_{T}$ events, the first two large- $R$ jets always contain the top quarks. At large $p_{T}$, the difference between $m_{T}$ and $p_{T}$ ordering is immaterial.

Given the output of Algorithm 1 for a specific event, we then identify the topology as follows:

- If each of $J_{1}$ and $J_{2}$ contains one top (anti-)quark, we declare the event topology to be FCR.

- If one of $J_{1}$ and $J_{2}$ contains a single top, and the other does not contain a top, we declare the event topology to be FEX.

- If one of $J_{1}$ and $J_{2}$ contains both tops, we declare the event topology to be GSP.

- Otherwise, we define the event topology as "other". 
One advantage of this simple approach is that it is straightforward to implement and gives a definite answer about the topology of each event.

\subsection{Results}

Let us now examine what happens when we analyse $t \bar{t}$ events according to the procedure outlined so far. We will consider events at a $p p$ centre-of-mass energy of $\sqrt{s}=13 \mathrm{TeV}$, simulated with the hvq process [46] of POWHEG Box v2 [47], revision $3660,{ }^{10}$ using the PDF4LHC15_nnlo_mc PDF set [28] via LHAPDF [48], and a top mass of $173 \mathrm{GeV}$, interfaced to Pythia 8.240 [49], Monash13 tune [50], with multiple particle interactions and hadronisation turned off. All jet clustering is performed with FastJet [51], version 3.3.2. In the future it would also be interesting to carry out similar studies to NNLO accuracy [52-54], for example taking advantage of recent developments in combining parton showers and $t \bar{t}$ production at NNLO [55].

As inputs to Algorithm 1, we take the truth tops from the event record, together with all partons not coming from the top decay. Since the hvq process is NLO for $t \bar{t}$ production, we expect to have FCR topologies accurate to NLO, and FEX and GSP accurate to LO, while other topologies are at best generated by the shower, so do not have any formal perturbative accuracy. For observables and topologies that start only at NLO $\left(\alpha_{s}^{3}\right)$, we have cross-checked the hvq results against the NLO calculation for $t \bar{t}+$ jet (i.e. up to $\alpha_{s}^{4}$ ) in its POWHEG implementation [56] and found good agreement.

In this section, even though we consider top quarks before their decay, we will still label one of them as leptonic and the other as hadronic. Explicit cross sections will include the branching ratio for one top to decay muonically and the other hadronically.

For each of the event-hardness characterisation scales from table 1, figure 2 shows the fractions of FCR, FEX and GSP, as a function of the hardness scale (other topologies are negligible). The expectations from table 3 for the dominant topologies at high scales are given on each plot. Those expectations are all well borne out for sufficiently hard events, $V \gtrsim 2 m_{\text {top }}$, and it striking to what extent different event-hardness characterisation scale choices lead to very different proportions of the various topologies. In particular, the simple analysis of table 2, which suggested that all three topologies could potentially be of the same size, is clearly reflected in the plots for the two hardness scales that are insensitive to

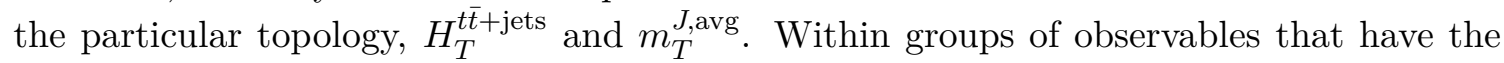
same dominant topologies according to table 3, there remain some differences. For example, $H_{T}^{t \bar{t}+\text { jets }}$ has a larger FEX component than does $m_{T}^{J, \text { avg }}$. This is not surprising, because the $H_{T}^{t \bar{t}+\text { jets }}$ variable receives a contribution from the initial-state top that is present in FEX topologies, while $m_{T}^{J, \text { avg }}$ does not.

To help visualise the kinematic features of the events in each of the three main topologies, figure 3 shows the two-dimensional distribution of $d \sigma / d z d \Delta \phi_{t \bar{t}}$ for large $m_{T}^{J \text {,avg }}$ for each topology. Here, $z=m_{T}^{\text {top, min }} / m_{T}^{J \text {,avg }}$ measures the hardness of the softer top relative to the underlying $2 \rightarrow 2$ event hardness, and $\Delta \phi_{t \bar{t}}$ is the azimuthal distance between the

\footnotetext{
${ }^{10}$ The renormalisation and factorisation scale choice is the standard choice of the hvq process, $m_{T}^{t}$ of the underlying Born configuration.
} 

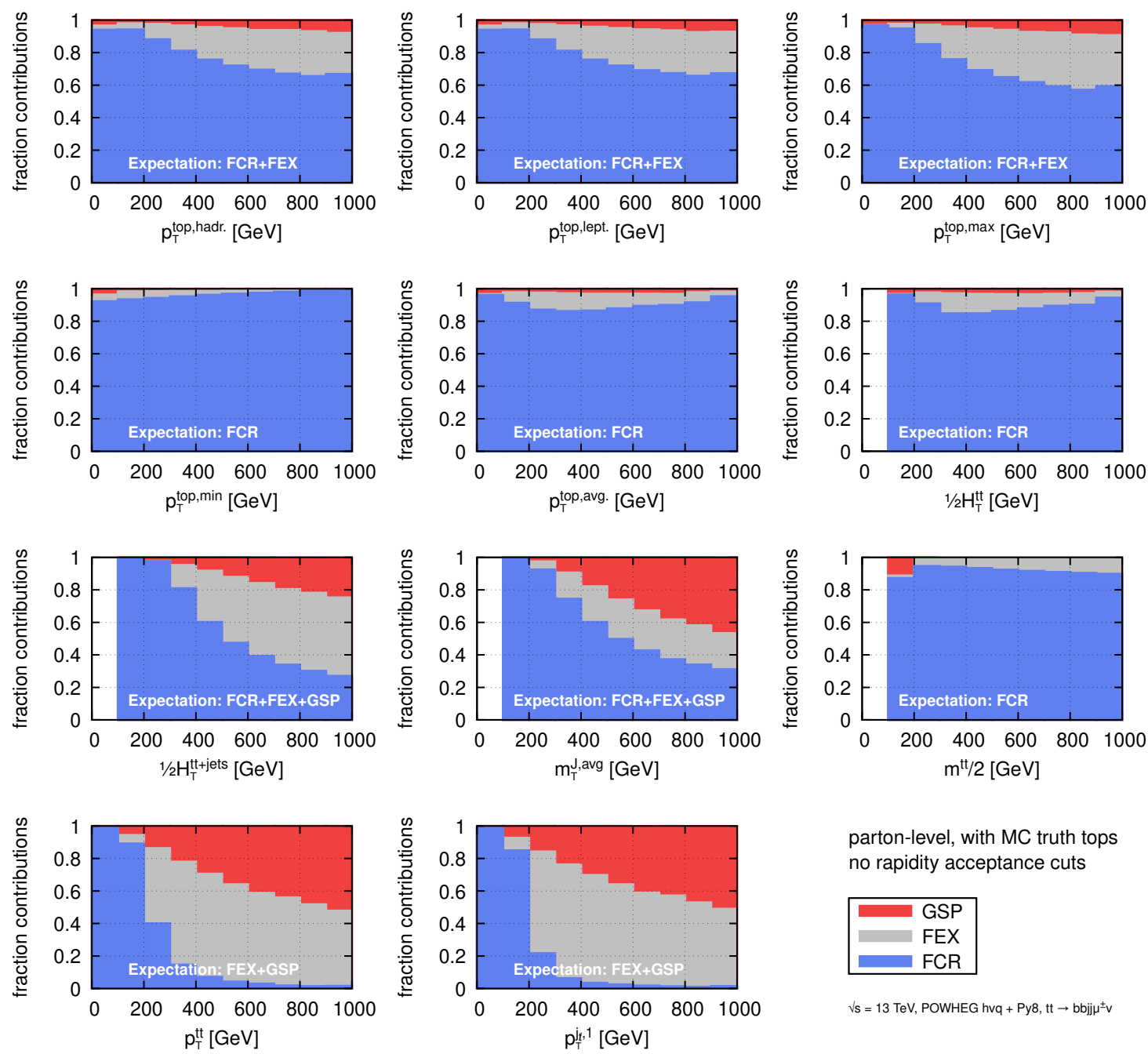

parton-level, with MC truth tops no rapidity acceptance cuts

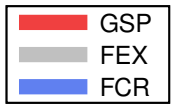

$\checkmark s=13$ TeV, POWHEG hvq + Py8, $t \mathrm{tt} \rightarrow$ bbjjp $\mu^{ \pm}$

Figure 2. Fractional contributions of the main top-production topologies (cf. figure 1), as a function of the variable used to characterise the hardness of the event, cf. table 1 . The expectations are those shown in table 3 .

two top quarks. In FCR topologies we expect the softer top to balance against the harder top $(z=1)$ and to be back-to-back in azimuth $\left(\Delta \phi_{t \bar{t}}=\pi\right)$. In FEX topologies, because the softer top is produced through initial-state radiation, we expect $z$ to take on predominantly low values and $\Delta \phi_{t \bar{t}}$ to be spread out between 0 and $\pi$. In GSP topologies, where the softer top is in the same jet as the harder top, we expect $z \lesssim 1 / 2$ and $\Delta \phi_{t \bar{t}}<R_{J}=1$. These features are broadly observed in the plots, though for the finite values of $m_{T}^{J \text {,avg }}$ that we use, the exact limits on $z$ are affected by the contributions of the top-quark mass to the variables that enter its definition.

Let us now apply the understanding that we have obtained to investigate differential cross sections that are commonly studied experimentally. Figure 4 shows differential cross sections for a subset of observables, choosing at least one from each of the groupings of 

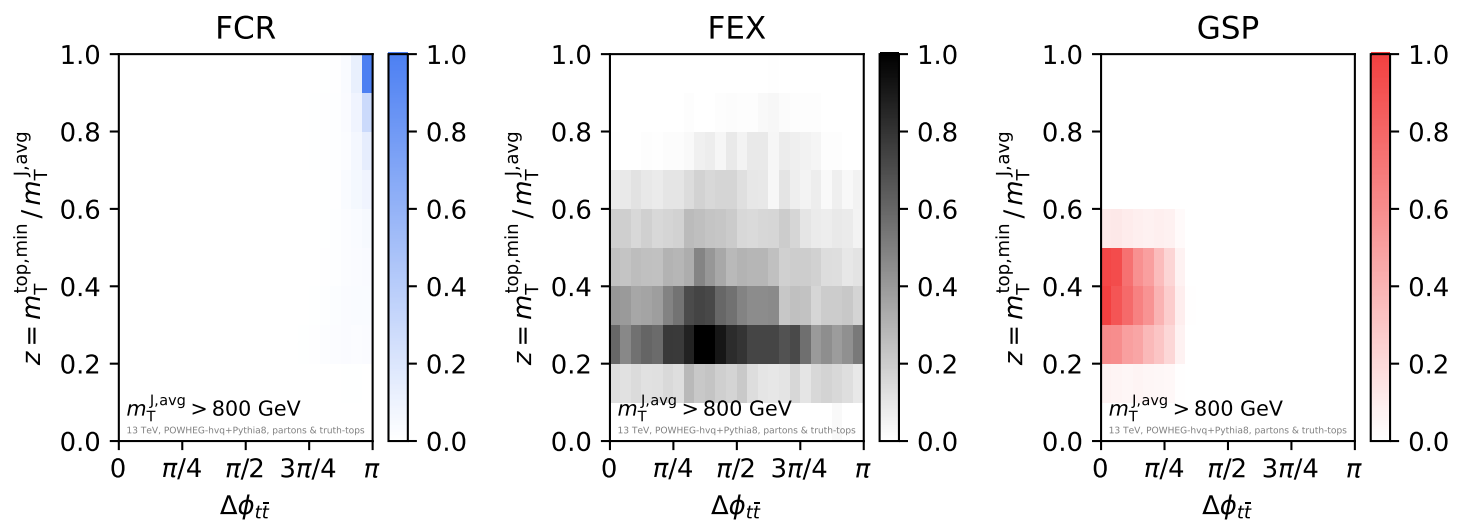

Figure 3. Kinematic distribution of the softer top, for events with large $m_{T}^{J, \text { avg }}$, in each of the three main topologies. The plots show $d \sigma / d z d \Delta \phi_{t \bar{t}}$, normalised to 1 at the maximum value of the histogram; $z=m_{T}^{\text {top, min }} / m_{T}^{J, \text { avg }}$ measures the hardness of the softer top relative to the underlying $2 \rightarrow 2$ event hardness, and $\Delta \phi_{t \bar{t}}$ is the azimuthal distance between the two top quarks.
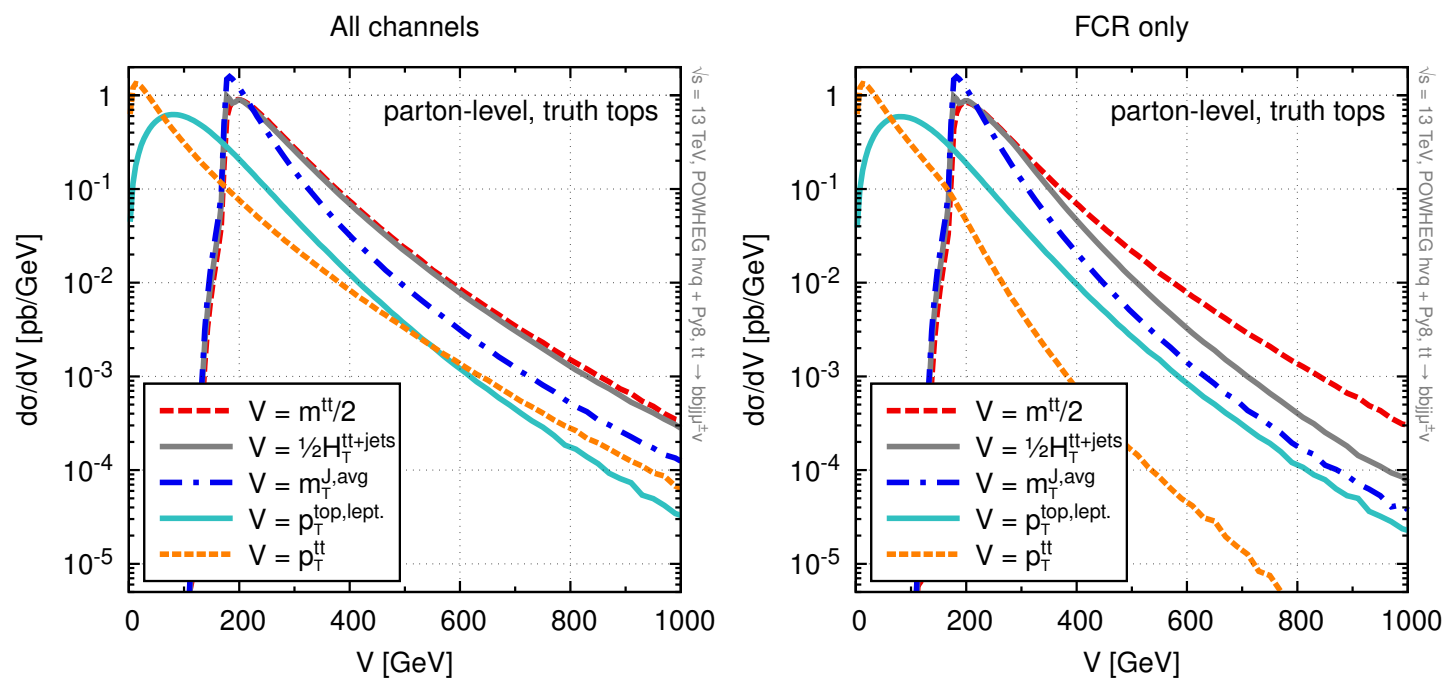

Figure 4. Differential cross sections as a function of a variety of variables used to characterise the event hardness $(V)$, considering an illustrative subset of the variables from table 1. Left-hand plot: results summing over all topologies. Right-hand plot: results for just the FCR topology.

table 1. The left-hand plot shows the results without any topological classification. Among the features in the plot that is surprising at first sight is that the $p_{T}^{t \bar{t}}$ distribution, which starts at $\alpha_{s}^{3}$, is larger at high scales than the $p_{T}^{\text {top,lep }}$ distribution, which starts at $\alpha_{s}^{2}$. Based on the analysis of section 2, this is however not a surprise, because of large FEX and GSP contributions to the $p_{T}^{t \bar{t}}$ distribution. If one considers only events with an FCR topology, as done in the right-hand plot, the $p_{T}^{t \bar{t}}$ distribution ends up being substantially suppressed relative to $p_{T}^{\text {top,lep }}$, restoring faith in an analysis based on perturbation theory.

Another feature that becomes clearer when isolating the FCR topology is the hierarchy between $\frac{1}{2} m^{t \bar{t}}$ on one hand and $H_{T}^{t \bar{t}+\text { jets }}, m_{T}^{J, \text { avg }}$ and $p_{T}^{\text {top,lep }}$ on the other. A hierarchy 

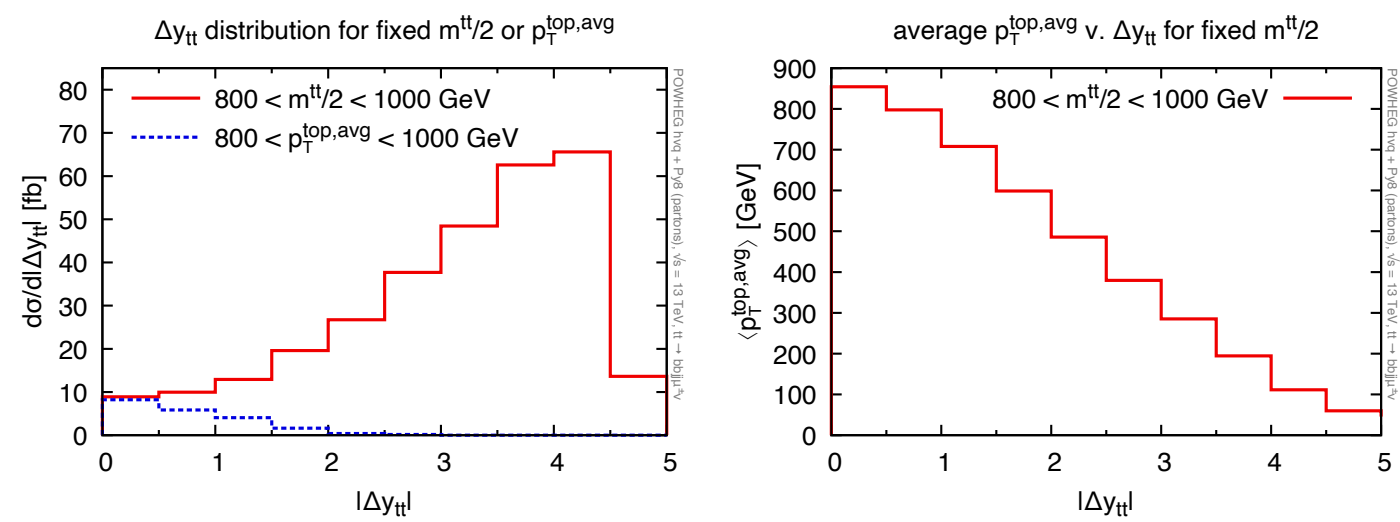

Figure 5. Left: the distribution of $\left|\Delta y_{t}\right|$ in for events where the $t \bar{t}$ pair is in a given bin of either $m^{t \bar{t}} / 2$ or $p_{T}^{\text {top,avg. }}$. Right: the average of the two top transverse momenta versus $\left|\Delta y_{t \bar{t}}\right|$ in the given bin of $m^{t \bar{t}} / 2$. We include all topologies in these results, keeping in mind that FCR is always the dominant contribution here.

is an expected consequence of the LO $\log \left(m^{t \bar{t}} / m_{\mathrm{top}}\right)$ enhancement for the $\frac{1}{2} m^{t \bar{t}}$ distribution quoted in eq. (2.3). The other three observables are identical at LO, and free of any $\log \left(m^{t \bar{t}} / m_{\text {top }}\right)$ enhancement. Yet in figure 4 (left) $H_{T}^{t \bar{t}+\text { jets }}$ appears to be almost identical to $\frac{1}{2} m^{t \bar{t}}$, and there is a clear hierarchy among $H_{T}^{t \bar{t}+\text { jets }}, m_{T}^{J \text {,avg }}$ and $p_{T}^{\text {top } \text {,lep }}$. If instead we examine figure 4 (right), with just the FCR topologies, the pattern is closer to the picture expected from LO: $\frac{1}{2} m^{t \bar{t}}$ is well above the other observables, with a relative enhancement that increases towards larger $\frac{1}{2} m^{t \bar{t}}$. Meanwhile, $H_{T}^{t \bar{t}+\text { jets }}, m_{T}^{J \text {,avg }}$ and $p_{T}^{\text {top,lep }}$ all show similar scaling at high momenta. Remaining variations between them are straightforward to understand: taking $m_{T}^{J \text { avg }}$ as the reference, the $H_{T}^{t \bar{t}+\text { jets }}$ variable includes contributions from ISR radiation and so is larger, while $p_{T}^{\text {top,lep }}$ is sensitive to the loss of radiation from top fragmentation and so is smaller. ${ }^{11}$

We have also checked the six other event-hardness scales from table 1 and the patterns observed are in line with the analysis given above.

Our final comment of this section concerns the observation that the $V=m^{t \bar{t}} / 2$ distribution is $12-14$ times larger than the $p_{T}^{\text {top,lep }}$ distribution. A significant ratio is expected because of the $\log \left(m^{t \bar{t}} / m_{\text {top }}\right)$ enhancement that is present in eq. (2.3), associated the integral over $\Delta y_{t \bar{t}}$ up to its kinematic boundary, eq. (2.4). Figure 5 (left) shows the $\Delta y_{t \bar{t}}$ distribution in a bin of either the $m^{t \bar{t}} / 2$ or $p_{T}^{\text {top,avg }}$ hardness scale. In the lowest bin of $\Delta y_{t \bar{t}}$, the results are independent of the choice of hardness scale. However at larger $\Delta y_{t \bar{t}}$, the difference between the two histograms is striking, with the $m^{t \bar{t}} / 2$ case dominated by values of $\Delta y_{t \bar{t}}$ close to the kinematic limit, a consequence not just of the LO distribution covering rapidities up to the kinematic boundary, but also of further apparent logarithmic

\footnotetext{
${ }^{11}$ Specifically, taking the example of $H_{T}^{t} \bar{t}+$ jets and recalling that one has a steeply falling cross section, a relatively small increase in $H_{T}^{t \bar{t}+\text { jets }}$ from a semi-soft jet can lead to a substantial increase in the cross section. In the case of $p_{T}^{\text {top,lep }}$, the effects of loss of $p_{T}$ to jet radiation are well known from corresponding studies of high- $p_{T} b$ production [73] and have been studied for top production in ref. [74].
} 
enhancements for large $\Delta y_{t \bar{t}}$ at NLO and beyond (cf. appendix B). It is important to be aware that the events at large $\Delta y_{t \bar{t}}$ involve low transverse momenta for the top quarks. This is illustrated in figure 5 (right), which shows the average top-quark transverse momentum as a function of $\Delta y_{t \bar{t}}$ for the same bin of $m^{t \bar{t}} / 2$ as shown on the left. Close to the kinematic boundary of $\Delta y_{t \bar{t}}$, where the cross section is largest, the top quarks have transverse momenta of the order of $m_{\text {top }}$, which is to be expected given the basic kinematic relations that hold at LO.

\section{Particle-level (reconstructed top) analysis}

In this section, we examine whether it is feasible to carry out an analysis of $t \bar{t}$ topologies in actual collider events. Standard experimental $t \bar{t}$ analyses take either a resolved approach, or a boosted approach [57-59] to identifying the top-quark decay products. However, this strategy breaks down for the FEX topology because one top is boosted, while the other may have only a moderate $p_{T}$. It breaks down also for the GSP topology, because a single fat jet contains both a top and an anti-top, and typical boosted-top tagging algorithms are not designed to identify both tops. Finally, we have seen that even in FCR topologies, a single bin of $m^{t \bar{t}} / 2 \gg m_{\text {top }}$ receives contributions from tops at low as well as high $p_{T}$, and so it is not sufficient to only apply boosted-top tagging algorithms in measurements of the (high) $m^{t \bar{t}} / 2$ distribution.

The strategy that we develop here is to adopt a resolved top identification algorithm as our baseline, but to provide inputs such that the algorithm continues to function even for tops at high $p_{T}$. We will restrict our study to semi-leptonic $t \bar{t}$ events, and work with the assumption that in a full experimental environment, lepton tagging, double $b$-tagging and a missing-energy threshold would be sufficient to reduce backgrounds to a manageable level. ${ }^{12}$

\subsection{Event analysis}

The event analysis algorithm that we develop as a proof of concept is given as Algorithm 2. It is intended to function across the full range of top transverse momenta with large LHC statistics, i.e. from low $p_{T}$ up to $p_{T} \simeq 1 \mathrm{TeV}$, and to be capable of reconstructing $t \bar{t}$ pairs that would lie in a single large- $R$ jet, thus addressing the issues raised in the introduction to this section. Some of the analysis steps involve a certain amount of choice. When choosing between methods that are best at very high $p_{T}$ (several times the top mass) and methods that are simple, we have generally chosen the latter.

Note that the declustering of step 3 is essential when the hadronic top quark is at high $p_{T}$, because it resolves the decay products even when they have been clustered into fewer than three $R=0.4$ jets $j{ }^{13}$ The approach that we use is similar to the early jet substructure work of Seymour [62] and also coincides with the approach adopted in the proposal [63] to use tops to characterise the time evolution of the heavy-ion medium. If one were studying top quarks with very high $p_{T}$, it would probably be better to develop

\footnotetext{
${ }^{12}$ We also ignore subtleties related to lepton identification in boosted top decays, see e.g. ref. [60].

${ }^{13}$ The declustering starts to have a significant impact on the reconstruction efficiency for $p_{T}^{\text {top, had }} \gtrsim$ $500 \mathrm{GeV}$.
} 


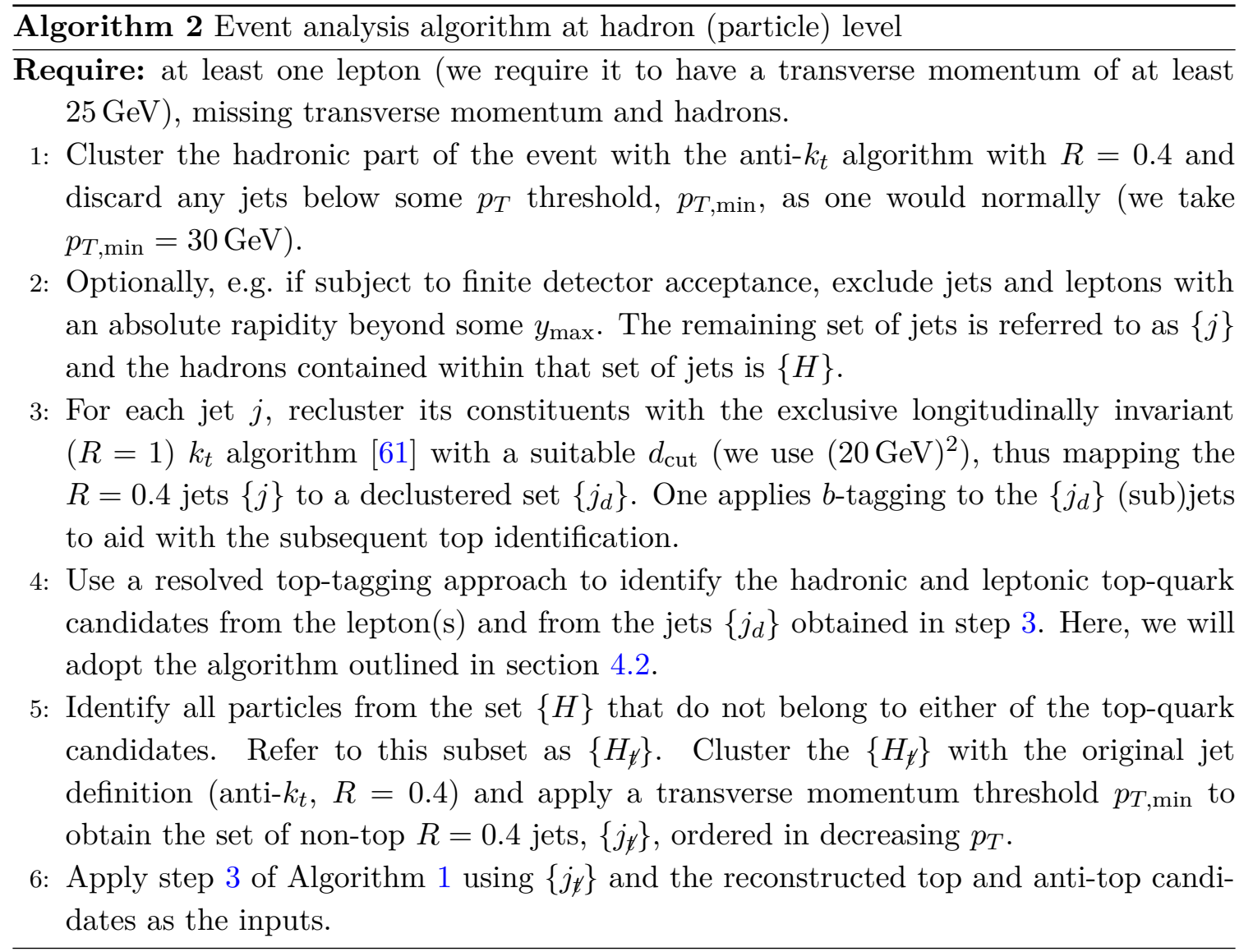

an approach based on the Cambridge/Aachen algorithm so as to reduce the high- $p_{T}$ toptagging's sensitivity to the underlying event and pileup. One might also wish to impose a constraint on the separation between candidate top decay products that depends on the $p_{T}$ of the top itself, similar to the variable- $R$ approach used in refs. [64, 65] or alternatively a kinematically analogous fractional momentum cut on individual prongs, as used in Soft Drop $[66,67]$ and a range of other taggers.

The choice to proceed via the $\left\{j_{\not}\right\}$ set in step 5 is motivated in particular if one wishes to compare $R=0.4$ jet observables with purely resolved measurements in the literature.

At very high $p_{T}$, instead of the $R_{J}=1$ anti- $k_{T}$ algorithm used in step 3 of Algorithm 1 , it might make more sense to adopt an algorithm such as flavour- $k_{T}[38,68]$ and possibly apply it directly to the hadrons $\left\{H_{\not t}\right\}$ and tops, i.e. to the set $\left\{H_{\not}, t, \bar{t}\right\}$. The flavour- $k_{t}$ algorithm suppresses the clustering of lone soft-quarks within a hard jet, a situation which would contaminate the flavour of a hard jet. ${ }^{14}$

\footnotetext{
${ }^{14}$ These configurations should be assigned to the "other" category of figure 1 , and this does not always occur with the anti- $k_{T}$ algorithm. The effects start only at order $\alpha_{s}^{2} \ln p_{T} / m_{\text {top }}$ relative to LO, and are practically negligible at the $p_{T}$ values that we study here, hence our choice to retain the simplicity of the anti- $k_{T}$ algorithm. The effects are conceptually interesting when $L=\ln p_{T} / m_{\text {top }} \gg 1$, because higher-order logarithms have a BFKL [69, 70] structure, as pointed out by Marchesini and Mueller [71].
} 


\subsection{Top reconstruction}

The top reconstruction that we use is a so-called "resolved" algorithm, i.e. one that takes advantage of the fact that the top decay products should map to separate jets. The declustering procedure in step 3 of Algorithm 2 helps ensure that this is true even for high- $p_{T}$ tops.

There are many resolved top reconstruction algorithms in use for semi-leptonic $t \bar{t}$ events, i.e. those with a lepton, missing energy and jets, some of them $b$-tagged. The procedure we adopt is largely based on the fiducial top definition proposed in ref. [72] and it makes use of invariant masses in order to choose which jets to cluster together to form top candidates. Our version has one small modification concerning the neutrino treatment.

First we reconstruct the neutrino momentum from the missing transverse momentum $p_{T}^{\text {miss }}$ and the lepton 4-momentum $p^{\text {lep }}$, setting $\vec{p}_{T}^{\nu}=\vec{p}_{T}^{\text {miss }}$ and determining $p_{z}^{\nu}$ from the constraint

$$
M\left(\nu_{l}+l\right)=m_{W}
$$

where $M(X)$ refers to the invariant mass of an object $X$ and $m_{W}$ is the mass of the $W$ boson. Solving the resulting quadratic equation generally presents us with two solutions for $p_{z}^{\nu}$, the component of the neutrino's momentum along the beamline. In cases where the two solutions are complex, we take their real part as the physical $p_{z}^{\nu}$, and where both are real we take the root with smaller $\left|p_{z}^{\nu}\right|$ (this is the one small point where we differ from ref. [72], which takes the root with larger $\left.\left|p_{z}^{\nu}\right|\right){ }^{15}$

With this estimate of the kinematics of the leptonically decaying $\mathrm{W}$ boson, we attempt to identify a subset of the reclustered (sub)jets $\left\{j_{d}\right\}$ with the remaining decay products. Defining a semi-leptonic $t \bar{t}$ pair candidate by assigning one $b$-tagged jet to the leptonically decaying top quark candidate $\left(t_{l}\right)$, another to the hadronically decaying top candidate $\left(t_{h}\right)$, and a pair of non $b$-tagged jets as the decay products of the hadronically decaying $W$ boson candidate $\left(W_{h}\right)$, we calculate the quantity

$$
K^{2}=\left[M\left(t_{h}\right)-m_{\mathrm{top}}\right]^{2}+\left[M\left(t_{l}\right)-m_{\mathrm{top}}\right]^{2}+\left[M\left(W_{h}\right)-m_{W}\right]^{2}
$$

for each combination of jets satisfying $140 \mathrm{GeV} \leq M\left(t_{h / l}\right) \leq 190 \mathrm{GeV}$, where $m_{\text {top }}=$ $173 \mathrm{GeV}$ is our top-quark mass choice (we do not place any direct requirements on $M\left(W_{h}\right)$ ). If no such combination of jets exists then we deem the reconstruction to have failed, otherwise the $t \bar{t}$ candidate pair with the lowest $K^{2}$ is taken to most closely describe the kinematics of the full decay chain.

\subsection{Validation of reconstruction performance}

To verify the performance of our reconstruction approach we consider events at parton level, where we include the decays of the top quarks and their subsequent showering. The use of

\footnotetext{
${ }^{15} \mathrm{~A}$ criterion for deciding on the strategy is whether one expects the top quarks to be predominantly at central rapidities (for which one would favour the root with smaller $\left|p_{z}^{\nu}\right|$ ), or predominantly at large rapidities (favouring the root with larger $\left|p_{z}^{\nu}\right|$ ). For all observables except $\frac{1}{2} m^{t \bar{t}}$, we expect the tops to be central, hence our choice. Having said that, the difference in the results is expected to be relatively small between the two choices.
} 

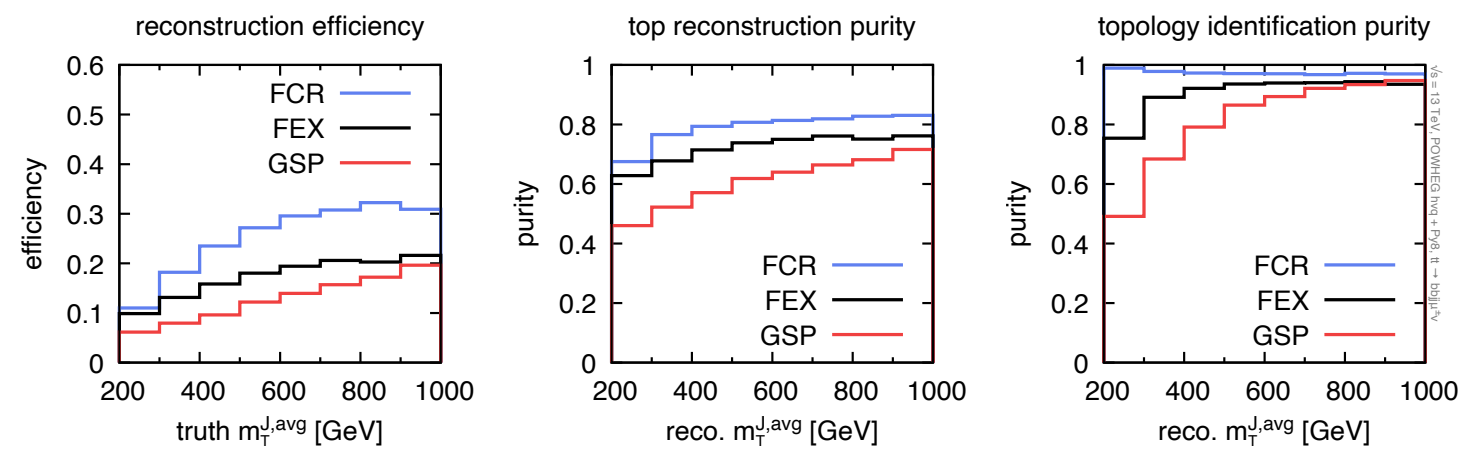

Figure 6. Tests of the efficiency and purity of the top reconstruction and topology identification procedures. See text for details.

parton-level events here allows us to unambiguously identify the source of each particle in the event, for example whether it came from a $W$ decay. We will show results as a function of $m_{T}^{J, \text { avg }}$, because this event hardness characterisation scale yields events with a mix of all three topologies, cf. figure 2.

Figure 6 (left) shows the overall efficiency of the top reconstruction and topology identification. Consider a topology that we wish to test, say FCR. We identify all events where, using the Monte Carlo truth top quarks, the topology was classified as FCR. For a given bin of Monte Carlo truth $m_{T}^{J \text {,avg }}$, we then determine the fraction of those events satisfying the following conditions:

1. the event analysis algorithm of section 4.1 and the top reconstruction algorithm of section 4.2 (using the final leptons and partons) should successfully identify hadronic and leptonic top candidates;

2. additionally the reconstructed top candidates should predominantly contain the corresponding truth top decay products, specifically, the $b$ quarks should be correctly assigned in each candidate, and the two jets that make up the $W$ candidate should each have received at least $50 \%$ of their $p_{T}$ from genuine $W$ decay products;

3. finally the event topology based on the reconstructed top quarks should also be FCR.

One sees that the efficiency is about $10 \%$ for low values of $m_{T}^{J \text { avg }}$, rising to $30 \%$ at large $m_{T}^{J \text { avg }}$, with the FEX and GSP efficiencies being slightly lower than for FCR, which is to be expected given that the FEX and GSP topologies are made more difficult to reconstruct by the lower transverse momenta and/or potential proximity of the top decay products.

We also verify the purity of the reconstruction, separating out the study of the purity of the top reconstruction and of the topology identification. Figure 6 (middle) shows the former. For a given reconstructed topology, it shows the fraction of the events in the given reconstructed $m_{T}^{J \text {,avg }}$ bin for which the reconstructed top candidates predominantly contain the corresponding truth top decay products (condition 2 above). The top purity is in the range $50-80 \%$, increasing with $m_{T}^{J \text {,avg }}$. Figure 6 (right) shows the purity for the topology identification. Here we consider all events reconstructed as being in a given topology, and 
examine the fraction for which the truth topology is the same as the reconstructed one (irrespective of the whether the top candidates match the truth ones). This purity rapidly tends to 1 with increasing $m_{T}^{J \text {,avg }}$.

Overall, the results of figure 6 give us confidence that the reconstruction approach proposed here can be successfully applied to realistic events.

\subsection{Results}

We close this article by repeating the main truth-level analyses of section 3.2 on hadronlevel events (with multi-parton interactions switched on), and imposing a realistic detector rapidity acceptance, i.e. considering only jets and muons at rapidities below 2.5 in step 2 of Algorithm 2.

Figure 7 is the analogue of figure 2 using top-quark candidates as reconstructed from particle (hadron) level events. The two sets of plots are strikingly similar, which should not be surprising given the validation results shown above. Where modest differences arise, these can be understood as a consequence of the variations in reconstruction efficiencies across different topologies. For example one sees a slightly larger FCR contribution in the hadron-level reconstructed $m_{T}^{J \text {,avg }}$ plot than in the parton-level one, reflecting the higher efficiencies for FCR reconstruction. Similarly we have checked that the hadron-level reconstructed analogue of figure 3 is close to the truth-level results.

Figure 8 shows hadron-level differential cross sections. Broadly speaking the results are similar to those with truth tops in figure 4 . There is an overall reduction in the cross sections, which is to be expected given the $10-30 \%$ reconstruction efficiencies shown in figure 6.

One additional striking difference is that the $m^{t \bar{t}} / 2$ distribution no longer shows the strong enhancement relative to other hardness scales at high values of $m^{t \bar{t}} / 2$, for example being only 4 times larger than the $p_{T}^{\text {top,lep }}$ distribution at around $1 \mathrm{TeV}$, rather than $12-14$ times larger in figure 4. This can be understood from figure 9, which compares results for $\Delta y_{t \bar{t}}$ at large $m^{t \bar{t}} / 2$ using truth tops and using tops as reconstructed from the final particles (in this case final-state hadrons). ${ }^{16}$ The reconstruction procedure has the largest impact at large values of $\Delta y_{t \bar{t}}$, where the top quarks have relatively low $p_{T}$ (which tends to reduce reconstruction efficiencies) and where additionally some of their decay products may fall beyond the rapidity acceptance. Figure 9 shows results for a current LHC acceptance of $|y|<2.5$ and for an HL-LHC type acceptance of $|y|<4$. The latter is almost identical to results with full rapidity acceptance. Since the enhancement of the truth $m^{t \bar{t}} / 2$ distribution is precisely due to the contributions from large $\Delta y_{t \bar{t}}$, it is now evident why that enhancement is reduced with the reconstructed tops in figure 8 .

Everything that we have seen so far about the $m^{t \bar{t}} / 2$ distribution suggests that considerable caution is warranted when predicting and measuring it. From a perturbative point

\footnotetext{
${ }^{16}$ For a related measurement see ref. [20]. The measurement is for a difference of top pseudorapidities, $\Delta \eta^{t \bar{t}}$, in a given bin of $m^{t \bar{t}}$, rather than a difference of rapidities, $\Delta y_{t \bar{t}}$. The features should broadly speaking be similar, though it is useful to be aware that $\Delta \eta^{t \bar{t}}$ is not invariant under longitudinal boosts, while $\Delta y_{t \bar{t}}$ is. Furthermore, for $p_{T}^{\text {top }} \lesssim m_{\text {top }}$ the pseudorapidity correlates less well with the pseudorapidities of the top decay products than does the rapidity of the top.
} 

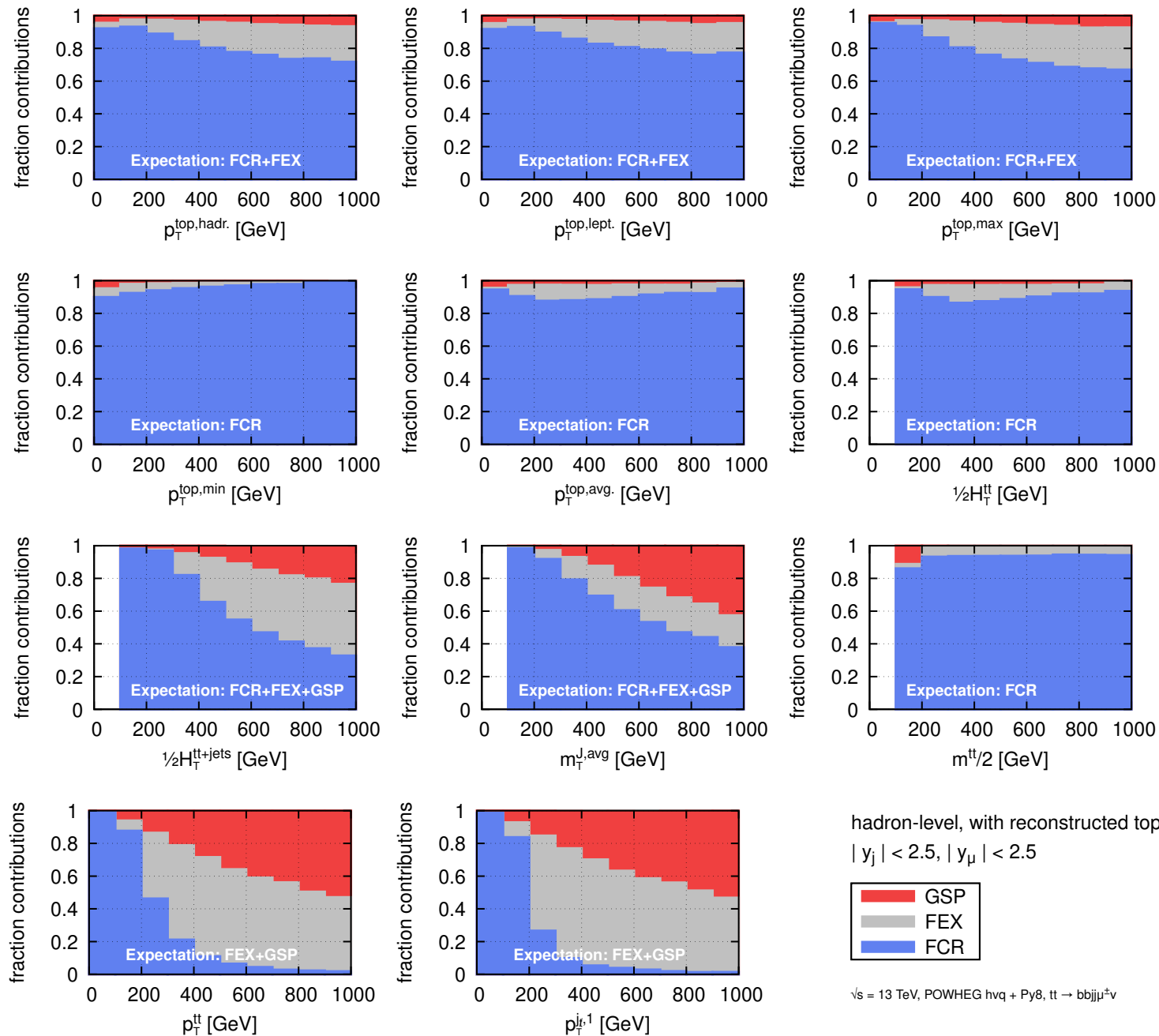

hadron-level, with reconstructed top $\left|y_{j}\right|<2.5,\left|y_{\mu}\right|<2.5$

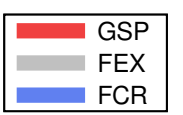

$V_{S}=13 \mathrm{TeV}$, POWHEG hvq + Py8, $\mathrm{tt} \rightarrow \mathrm{bbjj} \mu^{ \pm} \mathrm{v}$

Figure 7. Fractional contributions of the main top-production topologies as a function of the variable used to characterise the hardness of the event. This is the analogue of figure 2, replacing Monte-Carlo truth top quarks with top-quark candidates reconstructed in particle-level events using the approach of sections 4.1 and 4.2. A rapidity cut of 2.5 has been applied to the muons and to the $R=0.4$ jets obtained in step 1 of Algorithm 2 .

of view, the sensitivity to large $\Delta y_{t \bar{t}}$ separations brings concerns of logarithmic enhancements, as discussed in appendices A.1 and B (though further work is needed to quantify their impact, also considering different potential scale choices). From an experimental point view, a fiducial measurement of $m^{t \bar{t}} / 2$ should be robust, because it includes the acceptance, however an unfolded, top-parton level measurement would be subject to substantial unfolding corrections from the region of large $\left|\Delta y_{t \bar{t}}\right|$ where the acceptance is low. ${ }^{17}$ Overall,

\footnotetext{
${ }^{17}$ Schematically we envisage two approaches to the unfolding: one could measure the distribution differentially in bins of $\left|\Delta y_{t \bar{t}}\right|$ and divide each bin by its acceptance. This will result in division by an acceptance that is close to zero, which is dangerous. Alternatively, one could measure the fiducial cross section and multiply it by the ratio of total to fiducial cross sections. This is then very sensitive to the theoretical modelling of the dominant, large- $\left|\Delta y_{t \bar{t}}\right|$ region, which is again dangerous.
} 

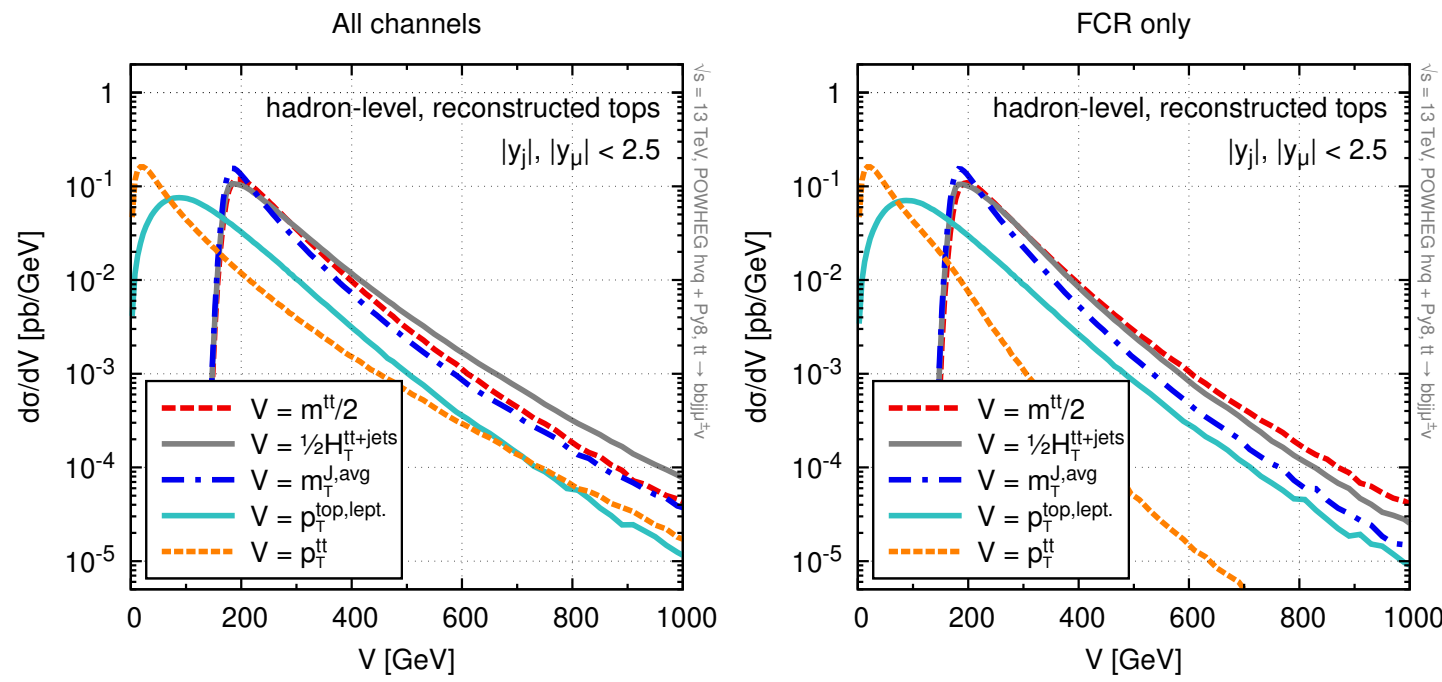

Figure 8. Hadron level results with reconstructed tops, for differential cross sections as a function of a selection of scales used to characterise the event hardness $(V)$, for a subset of such scales. Left-hand plot: results summing over all topologies. Right-hand plot: results for just the FCR topology. This plot is to be compared to figure 4. The results here include a rapidity acceptance cut of $|y|<2.5$ for jets and muons.

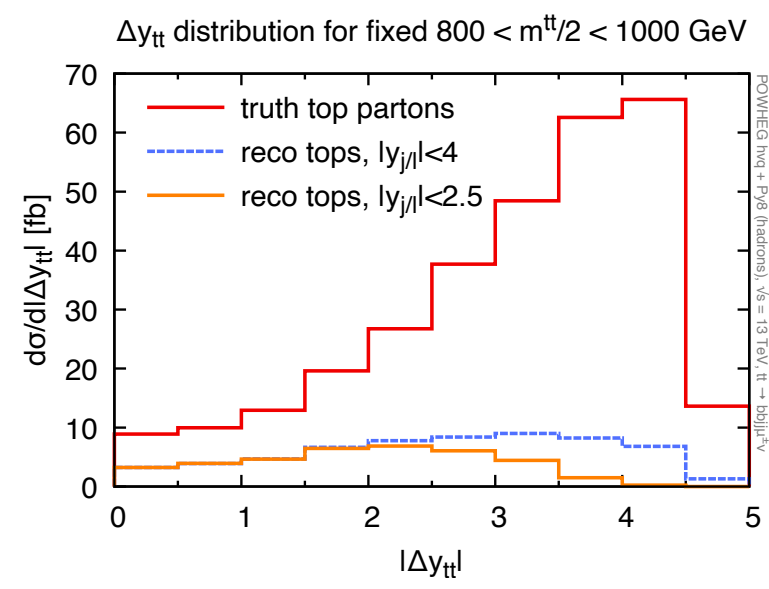

Figure 9. Comparison of the truth (partonic-top) $\Delta y_{t \bar{t}}$ distribution with the distribution obtained for fully reconstructed top quarks in hadron (particle) level events. The (truth or reconstructed) $t \bar{t}$ pair satisfies the constraint $800<m^{t \bar{t}} / 2<1000 \mathrm{GeV}$. The histograms include all topologies.

this suggests that for any kind of precision study with the $m^{t \bar{t}} / 2$ variable it would be wise to apply an upper limit on $\left|\Delta y_{t \bar{t}}\right|$, e.g. $\left|\Delta y_{t \bar{t}}\right|<2$. On one hand, within this region, figure 9 shows that measurements would then be largely unaffected by the experimental rapidity acceptance cuts on the input jets and leptons. On the other hand, the $\left|\Delta y_{t \bar{t}}\right|<2$ rapidity window is still large enough, for example, to exploit the different $\Delta y_{t \bar{t}}$ dependencies of the $q \bar{q}$ and $g g$-induced channels to separately constrain gluon and (anti)-quark PDFs, cf. figure 10 of appendix A.1. ${ }^{18}$

\footnotetext{
${ }^{18}$ Examination of figure 10 in appendix A.1 suggests that $\left|\Delta y_{t \bar{t}}\right|<2$ might also lessen the issues associated with choosing $\mu=\frac{1}{2} m_{t \bar{t}}$ for the $\frac{1}{2} m_{t \bar{t}}$ observable.
} 


\section{Conclusions}

The core observation of this paper is that energetic $t \bar{t}$ production involves far more than the simple leading-order back-to-back topology (flavour creation, FCR). If one selects events with a large momentum transfer and a $t \bar{t}$ pair, one finds roughly equal contributions from the FCR topology, and from each of the two topologies that start only at NLO: events with an initial-state $g \rightarrow t \bar{t}$ splitting (flavour excitation, FEX) followed by a hard scattering of one of the tops, and events with a non-top hard scattering that is followed by a final state $g \rightarrow t \bar{t}$ splitting (gluon splitting, GSP). The $H_{T}^{t \bar{t}+\text { jets }}$ and $m_{T}^{J \text {,avg }}$ panels of figure 2 illustrate that all three of these topologies have similar cross sections. The reason for this surprising pattern is that the underlying hard $2 \rightarrow 2$ scattering channels that dominate at NLO involve $t$-channel gluon exchange, and so have squared matrix elements that are an order of magnitude larger than for the LO scattering channels, which involves either an $s$-channel gluon exchange or a $t$-channel quark exchange. That enhancement numerically compensates for the extra factor of $\alpha_{s}$ that appears from the $g \rightarrow t \bar{t}$ splitting in the FEX and GSP channels, as can be seen from table 2. The specific mix of topologies depends critically on the choice of observable used to characterise the hardness of $t \bar{t}$ events, as anticipated in section 2.3 and verified figure 2. In particular, as one moves from event hardness variables that democratically measure the hardness across all event particles, towards variables that measure hardness specifically of the top-quarks, the fraction of the FCR contribution increases. For observables like $p_{T}^{\text {top,avg }}$, FCR comes to dominate.

An awareness of the role played by different topologies is important in experimental measurements. Identifying the top quarks in FEX and GSP topologies brings additional challenges as compared to the FCR topology, for example because the two tops may have significantly different transverse momenta, or because they may end up within a single jet. This requires the use of top reconstruction techniques that transcend the usual resolved versus boosted paradigm, and we proposed a suitable fiducial-top definition in section 4 , which combines the approaches of refs. [63] and [72]. The use of techniques that can successfully identify the $t \bar{t}$ pair in all topologies is especially critical for measurements that correct to the level of top-partons: if a measurement is blind to a certain topology, then the contribution from that topology to the final cross section risks being estimated entirely from simulation rather than data. ${ }^{19}$

As we saw in sections 3 and 4 it is possible to provide classifications of the topology of individual events. Given that these topologies involve different PDFs and different underlying $2 \rightarrow 2$ hard-scattering processes, separating out those topologies can help in extracting the most physics information from the data. This is potentially relevant in any use of energetic $t \bar{t}$ production for precision physics, whether PDF fits, EFT studies, searches for small direct BSM, or validation of simulation tools. A separation by topology would

\footnotetext{
${ }^{19}$ Similar issues with top reconstruction apply for the $m^{t \bar{t}}$ distribution, independently of any questions of topology, because at large $m^{t \bar{t}}$ the $t \bar{t}$ quarks themselves may both have low $p_{T}$ or high $p_{T}$, depending on their rapidity separation. Given that large rapidity separations tend to be difficult to reconstruct, because of finite detector acceptances, cf. figure 9, it might be advisable for future measurements of the $m^{t \bar{t}}$ distribution to include a cut on $\Delta y_{t \bar{t}}$, e.g. $\left|\Delta y_{t \bar{t}}\right|<2$, as discussed at the end of section 4.4 .
} 
also seem wise when studying the order-by-order convergence of perturbative predictions. We therefore encourage future measurements and theoretical studies to further explore the rich topological structure of energetic $t \bar{t}$ production.

One consideration that we have not explored in any depth is that of enhancements of perturbative contributions by logarithms of the hardness scale divided by the top mass (e.g. FONLL [73, 74], BFKL in both $t$-channel quark [75-78] and gluon exchange [69, 70], BFKL in EW exchange [79], double logarithms [80-83] and BFKL logarithms [71, 84, 85] in final state fragmentation, and double-log small- $x$ non-singlet enhancements $[86,87])$. At LHC energies, such logarithms only start to become relevant for $t \bar{t}$ production (cf. eq. (2.7)). However, theoretically, the breadth of different classes of logarithmic enhancement would make for a compelling study. Such a study would probably be called for at higher collider energies (e.g. the $100 \mathrm{TeV}$ of the FCC-hh), and could potentially also be of interest for $b \bar{b}$ production at the HL-LHC.

\section{Acknowledgments}

We are grateful to the authors of ref. [55] for supplying us with the MiNNLO event sample that we used for cross-checks in appendix B, to Pier Monni, Paolo Nason and Giulia Zanderighi for comments on the manuscript and to Lucian Harland-Lang and Gregory Soyez for helpful discussions. We also thank the referee for useful suggestions of clarifications of the manuscript. This work was supported by the Science and Technology Facilities Council (STFC) under grants ST/P000770/1 (FD) and ST/T000864/1 (FC and GPS), by a Royal Society Research Professorship (RP $\backslash \mathrm{R} 1 \backslash 180112)$ and by the European Research Council (ERC) under the European Union's Horizon 2020 research and innovation programme, grant agreements 804394 (HipQCD, FC) and 788223 (PanScales, GPS). RWM wishes to acknowledge the Rudolf Peierls Centre for Theoretical Physics for support in the context of the Undergraduate Research Opportunities Programme.

\section{A Leading-order distributions}

For reference, we present and comment on some analytic formulas for leading-order $t \bar{t}$ cross sections, in a limit where one kinematic variable is much larger than the top mass. The results are essentially textbook level, and can be straightforwardly be derived from matrix elements to be found, e.g., in ref. [35]. They help provide some of the background to section 2.1.

\section{A.1 Distributions differential in $m_{t \bar{t}}$}

While in the main text we have used $m^{t \bar{t}} / 2$ for distributions, to keep the notation more compact, here we use the $t \bar{t}$ mass rather than half the mass, and write it as $m_{t \bar{t}}$. We also write $m_{t} \equiv m_{\text {top }}$. We consider the region $m_{t \bar{t}} \gg m_{\text {top }}$. We start with a distribution that is double differential in $m_{t \bar{t}}$ and the rapidity between the top and anti-top quarks, 
$\Delta y_{t \bar{t}}=y_{t}-y_{\bar{t}}$

$\frac{d \sigma}{d m_{t \bar{t}}^{2} d \Delta y_{t \bar{t}}} \simeq \frac{\alpha_{s}^{2} \pi}{m_{t \bar{t}}^{4}}\left[\frac{\left(8 \cosh \Delta y_{t \bar{t}}-1\right) \cosh \Delta y_{t \bar{t}}}{48\left(1+\cosh \Delta y_{t \bar{t}}\right)^{2}} \mathcal{L}_{g g}\left(m_{t \bar{t}}^{2} / s\right)+\frac{2}{9} \frac{\cosh \Delta y_{t \bar{t}}}{\left(1+\cosh \Delta y_{t \bar{t}}\right)^{2}} \mathcal{L}_{q \bar{q}}\left(m_{t \bar{t}}^{2} / s\right)\right]$.

The partonic luminosities are defined as

$$
\mathcal{L}_{i j}(x)=\left(2-\delta_{i j}\right) \int_{x}^{1} \frac{d z}{z} z f_{i}(z) \frac{x}{z} f_{j}(x / z),
$$

where $z f_{i}(z)$ is the distribution of partons of flavour $i$ and momentum fraction $z$ and $\mathcal{L}_{q \bar{q}}$ sums over $q \bar{q}$ flavours. In eq. (A.1), we have neglected corrections of the form $m_{\mathrm{top}}^{2} /\left(m_{T}^{t}\right)^{2}$. For $\Delta y_{t \bar{t}} \lesssim 1$, the condition $m_{t \bar{t}} \gg m_{t}$ implies large $m_{T}^{t} \gg m_{t}$ and so they can be neglected. For large $\Delta y_{t \bar{t}}$, one can have $m_{T}^{t} \sim m_{t}$, however one can verify that the relative contribution of the $m_{t}^{2} /\left(m_{T}^{t}\right)^{2}$ terms is suppressed by a power of $\cosh \Delta y_{t \bar{t}}$.

In eq. (A.1), for large $\Delta y_{t \bar{t}}$, the term proportional to the gluon luminosity becomes independent of $\Delta y_{t \bar{t}}$, i.e. one obtains a flat distribution in $\Delta y_{t \bar{t}}$. In contrast, the term proportional to the quark-antiquark luminosity vanishes. This difference in behaviour is a consequence of the fact that the $g g$ channel includes a $t$-channel quark-exchange diagram, whereas the $q \bar{q}$ channel only involves $s$-channel exchanges.

The result in eq. (A.1) is valid up to the kinematic limit given in eq. (2.4), $\Delta y_{t \bar{t}}^{\max } \simeq$ $\ln \frac{m_{t \bar{t}}^{2}}{m_{t}^{2}}$. It is illustrated for fixed scale in the coupling and PDFs in figure 10 (left), separated into the $g g$ and $q \bar{q}$-induced components. However, it is physically inappropriate to use a fixed scale, even for a single value of $m_{t \bar{t}}$, because different rapidities involve substantially different transverse momenta, and associated momentum transfers. The impact of using a physically motivated scale choice, $\mu_{R}^{2}=\mu_{F}^{2}=\left(H_{T}^{t \bar{t}} / 2\right)^{2}=m_{t \bar{t}}^{2} /\left(2\left(1+\cosh \Delta y_{t \bar{t}}\right)\right)$, is shown in the right-hand plot. ${ }^{20}$ This choice has a major impact on the shape of the distribution, with the plateaus in the $g g$-induced distribution acquiring a strong quasi-linear dependence on $\Delta y_{t \bar{t}}$. This dependence arises from the scaling violations in the coupling and PDF, a consequence of $\ln \mu^{2} \simeq \ln m_{t \bar{t}}^{2}-\Delta y_{t \bar{t}}$. The precise slope depends on the $x$ values being probed in the PDF.

The significant difference in $\Delta y_{t \bar{t}}$ dependence for $q \bar{q}$ and $g g$-induced production has the potential to provide a valuable handle separately for the gluon and quark parton distributions.

We can also integrate over $\Delta y_{t \bar{t}}$ to obtain the single-differential distribution,

$$
\frac{d \sigma}{d m_{t \bar{t}}^{2}}=\frac{\alpha_{s}^{2} \pi}{m_{t \bar{t}}^{4}}\left[\left(\frac{1}{3} \ln \frac{m_{t \bar{t}}^{2}}{m_{t}^{2}}-\frac{7}{12}\right) \mathcal{L}_{g g}+\frac{8}{27} \mathcal{L}_{q \bar{q}}\right],
$$

quoted in section 2.1 as eq. (2.3). Again we have neglected corrections that are suppressed in our kinematic region. The result is obtained for a fixed scale and, as discussed above, this is a physically inappropriate choice. Nevertheless, it is instructive to have the analytical result in this limit, because it reveals a $\ln \frac{m_{t \bar{t}}^{2}}{m_{t}^{2}}$ enhancement of the gluon-induced contribution.

\footnotetext{
${ }^{20}$ For more complex events, one may choose to generalise this to $m_{T}^{J \text {,avg }}$ or $H_{T}^{t \bar{t}+\text { jets }} / 2$ of table 1 .
} 

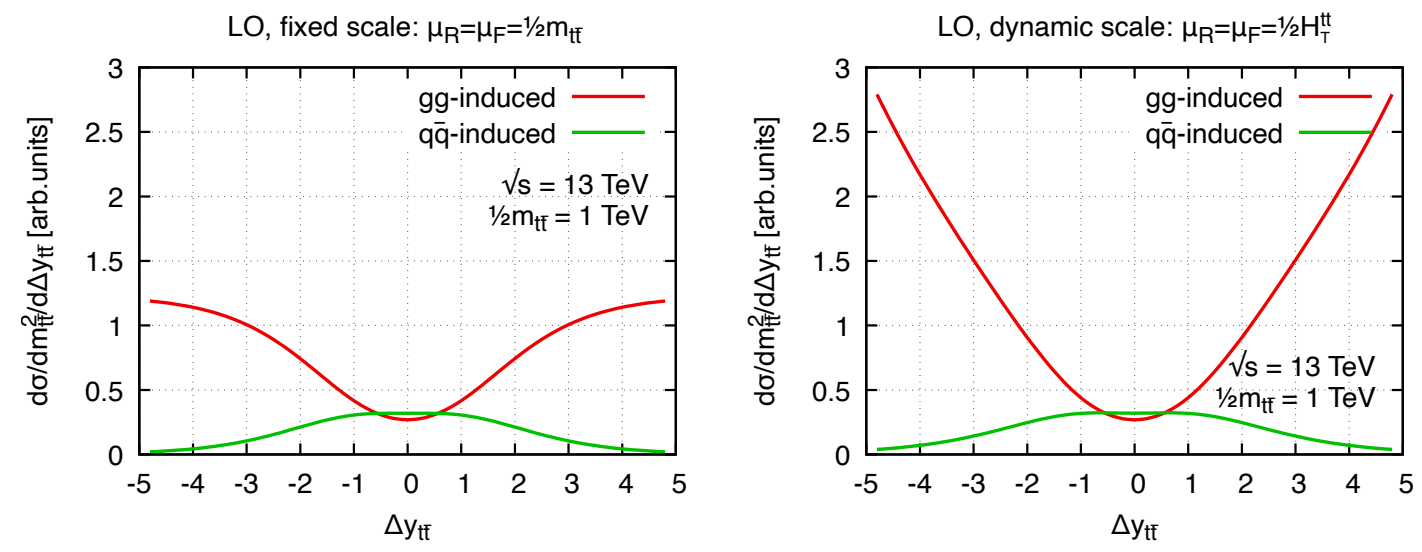

Figure 10. Asymptotic leading-order forms for the $\Delta y_{t \bar{t}}$ distribution at fixed large $m_{t \bar{t}}$, as given in eq. (A.1), applied to the case of $\sqrt{s}=13 \mathrm{TeV} p p$ collisions and $m_{t \bar{t}}=2 \mathrm{TeV}$. The left-hand plot uses fixed renormalisation and factorisation scales, which is physically inappropriate but illustrates the key analytical features of the structure of eq. (A.1). The right-hand plot uses a physically motivated scale choice, of the order of the momentum transfers involved in the process.

\section{A.2 Distributions differential in the top transverse momentum}

For large $p_{T, t} \gg m_{t}$, the leading order top-quark distribution doubly-differential in $p_{T, t}$ and $\Delta y_{t \bar{t}}$ is given by

$$
\begin{aligned}
\frac{d \sigma}{d p_{T, t}^{2} d \Delta y_{t \bar{t}}} \simeq & \frac{\alpha_{s}^{2} \pi}{4 p_{T, t}^{4}}\left[\frac{\left(8 \cosh \Delta y_{t \bar{t}}-1\right) \cosh \Delta y_{t \bar{t}}}{24\left(1+\cosh \Delta y_{t \bar{t}}\right)^{3}} \mathcal{L}_{g g}\left(2\left(1+\cosh \Delta y_{t \bar{t}}\right) p_{T, t}^{2} / s\right)+\right. \\
& \left.+\frac{4}{9} \frac{\cosh \Delta y_{t \bar{t}}}{\left(1+\cosh \Delta y_{t \bar{t}}\right)^{3}} \mathcal{L}_{q \bar{q}}\left(2\left(1+\cosh \Delta y_{t \bar{t}}\right) p_{T, t}^{2} / s\right)\right]
\end{aligned}
$$

neglecting terms relatively suppressed by powers of $m_{t}^{2} / p_{T, t}^{2}$. The structure here is very similar to that of eq. (A.1), and indeed it is a trivial rewriting of that result since at LO any combination of $p_{T, t}$ and $\Delta y_{t \bar{t}}$ can be mapped to a combination of $m_{t \bar{t}}$ and $\Delta y_{t \bar{t}}$. Still, some features change: we have an overall factor of $1 / p_{T, t}^{4}$ rather than $1 / m_{t \bar{t}}^{4}$; inside the outer square bracket the denominators have three powers of $\left(1+\cosh \Delta y_{t \bar{t}}\right)$ rather than two. These are trivial consequences of the LO relation between $m_{t \bar{t}}$ and $p_{T, t}$, and of the Jacobian transformation in the differential cross section. Importantly, for a given bin of $p_{T, t}$ the luminosities are evaluated at a mass that now depends on the rapidity separation, whereas in a given bin of $m_{t \bar{t}}$ they didn't.

The distributions for the rapidity difference are illustrated in figure 11. While the $g g$ induced term still has a broader $\Delta y_{t \bar{t}}$ distribution than the $q \bar{q}$-induced term, the difference in shapes is much smaller than for fixed $m_{t \bar{t}}$. In particular, both distributions are now concentrated around $\Delta y_{t \bar{t}}=0$ and quite strongly peaked there: this is partly because of the extra power of $\left(1+\cosh \Delta y_{t \bar{t}}\right)$, and partly because the partonic luminosities drop off rapidly with increasing rapidity separation. The kinematic limit at fixed $p_{T, t}$ is reached 

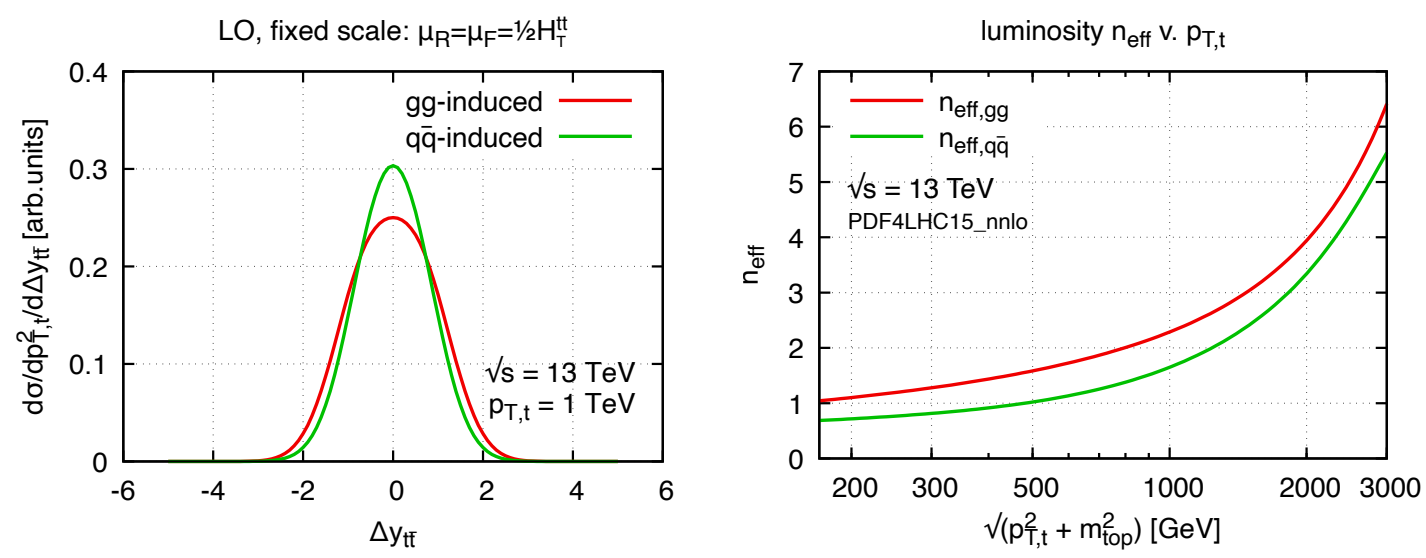

Figure 11. Left: asymptotic leading-order forms for the $\Delta y_{t \bar{t}}$ distribution at fixed large $p_{T, t}$, as given in eq. (A.4), applied to the case of $\sqrt{s}=13 \mathrm{TeV} p p$ collisions and $p_{T, t}=1 \mathrm{TeV}$. Right: effective power-law dependence, $n_{\text {eff }}$ of the partonic luminosities, as a function of $H_{T}^{t \bar{t}} / 2=\sqrt{p_{T, t}^{2}+m_{\mathrm{top}}^{2}}$. See text for details.

when $2\left(m_{t}^{2}+p_{T, t}^{2}\right)\left(1+\cosh \Delta y_{t \bar{t}}\right)=s$, giving

$$
\Delta y_{t \bar{t}}^{\max } \mid p_{T, t}=\cosh ^{-1}\left(\frac{s}{2\left(m_{t}^{2}+p_{T, t}^{2}\right)}-1\right) \simeq \ln \frac{s}{p_{T, t}^{2}},
$$

where $s$ is the proton-proton centre-of-mass energy and the approximation in the right-hand answer is valid for $m_{t}^{2} \ll p_{T, t}^{2} \ll s$.

The dependence of the partonic luminosities in eq. (A.4) on the rapidity separation means that one cannot analytically integrate over $\Delta y_{t \bar{t}}$ to obtain $d \sigma / d p_{T, t}$. However, for the purpose of understanding the key structural elements we make the approximation that the luminosity scales with the system mass as a power law,

$$
\mathcal{L}\left(m^{2}\right) \propto\left(\frac{1}{m^{2}}\right)^{n_{\mathrm{eff}}} .
$$

Equivalently, one may define

$$
n_{\mathrm{eff}}=-\frac{d \ln \mathcal{L}\left(m^{2}\right)}{d \ln m^{2}} .
$$

The effective power $n_{\text {eff }}$ is shown in figure 11 (right) as a function of the top transverse mass (or equivalently, half the system mass for $\Delta y_{t \bar{t}}=0$ ). With this approximation, one can integrate eq. (A.4) over $\Delta y_{t \bar{t}}$ to obtain

$$
\frac{d \sigma}{d p_{T, t}^{2}} \simeq \frac{\alpha_{s}^{2} \pi}{4 p_{T, t}^{4}}\left[f_{g g}\left(n_{\mathrm{eff}, g g}\right) \mathcal{L}_{g g}\left(4 p_{T, t}^{2} / s\right)+f_{q \bar{q}}\left(n_{\mathrm{eff}, g g}\right) \mathcal{L}_{q \bar{q}}\left(4 p_{T, t}^{2} / s\right)\right]
$$

where $f_{g g}\left(n_{\mathrm{eff}, g g}\right)$ and $f_{q \bar{q}}\left(n_{\mathrm{eff}, g g}\right)$ are purely numerical factors, which can be expressed in terms of hypergeometric functions of $n_{\text {eff }}$, but are perhaps more usefully shown for specific values of $n_{\text {eff }}$, cf. table 4 .

The normalisations of eqs. (A.3) and (A.8) have been chosen such that the contents of the square brackets in each can be directly compared. Specifically, the relative sizes 


\begin{tabular}{|ccccc|}
\hline$n_{\text {eff }}$ & 1 & 2 & 3 & 4 \\
\hline$f_{g g}\left(n_{\text {eff }}\right)$ & $\frac{16}{105}$ & $\frac{34}{315}$ & $\frac{128}{1485}$ & $\frac{3296}{45045}$ \\
$f_{q \bar{q}}\left(n_{\text {eff }}\right)$ & $\frac{128}{945}$ & $\frac{64}{567}$ & $\frac{1024}{10395}$ & $\frac{1024}{11583}$ \\
\hline
\end{tabular}

Table 4. The coefficients that appear in eq. (A.8) for gluon-gluon and quark-antiquark collisions as a function of the effective power-law dependence, $n_{\mathrm{eff}}$, of the partonic luminosities on the system mass squared.
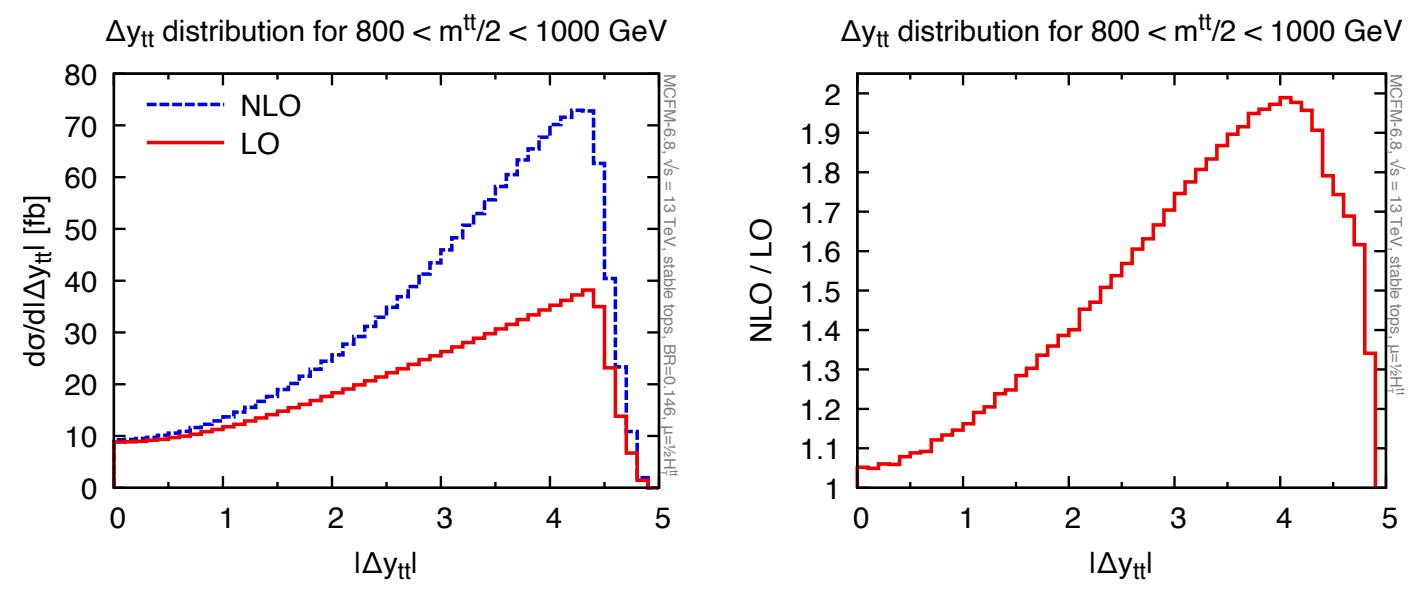

Figure 12. Left: NLO v. LO prediction for the $\Delta y_{t \bar{t}}$ distribution in a bin of large $m^{t \bar{t}} / 2$, as obtained with the MCFM program [88], including the semi-muonic branching fraction. The renormalisation and factorisation scales are set to $\mu=H_{T}^{t \bar{t}} / 2$. Right: corresponding K-factor, i.e. the ratio of the NLO to the LO result.

of the contents of the square brackets reflect the relative sizes at LO of the $d \sigma / d\left(\frac{1}{2} m_{t \bar{t}}\right)^{2}$ and $d \sigma / d\left(p_{T, t}\right)^{2}$ distributions. Taking the working point $\frac{1}{2} m_{t \bar{t}} \simeq p_{T, t} \simeq 1 \mathrm{TeV}$, where the $g g$ and $q \bar{q}$ partonic luminosities are comparable for $\sqrt{s}=13 \mathrm{TeV}$ (cf. table 2), and taking into account $n_{\text {eff }} \simeq 2$ from figure 11 (right), we see that the distribution of (half) the invariant mass is about six times larger than that for the transverse momentum of a top quark. Higher-order corrections will of course affect this result, but the basic pattern of a strong (logarithmic) enhancement for the $\frac{1}{2} m_{t \bar{t}}$ distribution relative to the $p_{T, t}$ distribution provides a helpful analytic explanation of the pattern seen in figure 4 .

\section{B Comments on the $m_{t \bar{t}}$ distribution beyond LO}

Figure 12 compares LO and NLO fixed-order calculations (obtained using MCFM [88]) with a renormalisation and factorisation scale choice of $H_{T}^{t \bar{t}} / 2$. This scale choice already absorbs the running coupling and PDF effects associated with the varying top transverse momentum across the range of $\Delta y_{t \bar{t}}$ values (cf. the left and right-hand plots of figure 10). Those effects correspond to an $\alpha_{s}^{3} \ln ^{2} m_{t \bar{t}} / m_{\text {top }}$ contribution to the total cross section, i.e. a single-logarithmic enhancement. However figure 12 shows further strong dependence of the NLO/LO $K$-factor as a function of $\Delta y_{t \bar{t}}$, which one may take evidence of further sources of logarithmic enhancement. 
A full discussion of the different potential sources of logarithmic enhancement is significantly beyond the scope of this paper. However, we believe that it is still informative to outline the different classes of term that can contribute. Loss of top momentum through fragmentation can contribute logarithms at small $\Delta y_{t \bar{t}}$ (i.e. large $p_{T}^{t}$ ), and is traditionally accounted for in the FONLL formalism [73, 74]. At large $\Delta y_{t \bar{t}}$, for $t$-channel top-quark exchange there are single logarithmic $t$-channel-fermion analogues of BFKL enhancement [75-78], associated with integrals over the rapidity of emitted gluons between the two final-state top quarks. Integrating over $\Delta y_{t \bar{t}}$, we also expect double logarithmic $\alpha_{s}^{n} \ln ^{2 n} m_{t \bar{t}} / m_{\text {top }}$ enhancements, which relate to double-logarithmic non-singlet structure functions at small- $x$ [86, 87], whose formalism can be used [89] to predict the $\alpha_{s}^{n} \ln ^{2 n-2} x$ terms in the non-singlet $P_{\mathrm{NS}}^{+}(x)$ splitting functions [30, 90, 91]. To understand their origin in the context of $t \bar{t}$ production, one may examine the NLO case: consider an ISR $g \rightarrow t \bar{t}$ splitting followed by a harder $t g \rightarrow g t$ scattering (which proceeds mainly through $t$-channel top exchange). We are interested in a situation where, in the ISR splitting, the anti-top and top have transverse momenta equal to some value $p_{t 1}$. Take the anti-top to be emitted into the final-state, and the top to be the particle that initiates the $t g \rightarrow g t$ hard scattering. The anti-top and top carry longitudinal momentum fractions $1-z$ and $z$ respectively, with $z \ll 1$. The $t g \rightarrow g t$ scattering itself involves large $s,|u| \gg|t| \gg p_{t 1}^{2}$. The $z P_{g \rightarrow t \bar{t}}(z)$ splitting function goes as $\alpha_{s} z$, and the reduced cross section $d \sigma / d \ln m_{t g}$ for the $t g \rightarrow g t$ scattering goes as $\frac{\alpha_{s}^{2}}{m_{t g}^{2}} \ln \frac{m_{t g}}{p_{t 1}}$, where $m_{t g}^{2}=z m_{t \bar{t}}^{2}$. The $z$ factor from the splitting function compensates the $1 / z$ from the $1 / m_{t g}^{2}=1 /\left(z m_{t \bar{t}}^{2}\right)$ factor in the reduced cross section, resulting in a logarithmic integral over $z$. There is also a logarithmic integral over $p_{t 1}$. Thus, after all integrations, we have a total cross section that goes as $\alpha_{s}^{3} \ln ^{3} m_{t \bar{t}} / m_{\text {top }}$, i.e. a double-logarithmic enhancement relative to the LO cross section.

Starting from NNLO, additional contributions arise that involve $t$-channel gluon exchange, associated with four-top production. At sufficiently large $m_{t \bar{t}}$ these will dominate the $m_{t \bar{t}}$ distribution because $d \sigma / d m_{t \bar{t}}^{2}$ scales as $\alpha_{s}^{4} /\left(m_{\text {top }}^{2} m_{t \bar{t}}^{2}\right)$ instead of the $\alpha_{s}^{2} / m_{t \bar{t}}^{4}$ seen in eq. (2.3). ${ }^{21}$ There are also electroweak contributions from $q \bar{q} \rightarrow t \bar{t}$ through a $t$-channel $W$ exchange, which is dominated by $q \bar{q}=b \bar{b}$ incoming flavours. These scale as $\alpha_{\mathrm{EW}}^{2} /\left(m_{\mathrm{top}}^{2} m_{t \bar{t}}^{2}\right)$, with a further $\alpha_{s}^{2} \ln ^{2} m_{\mathrm{top}} / m_{b}$ suppression factor coming from the requirements of two $b$-PDFs rather than gluon PDFs. These $t$-channel vector-boson exchange contributions, whether they involve gluons or electroweak bosons, will additionally be enhanced by QCD [69, 70] and EW [79] BFKL-type logarithms. We have verified the size of 4-top and EW processes using Alpgen [92] v. 2.14 and MadGraph5_aMC@NLO [93] v. 2.8 .2 respectively. At the LHC, even at large $m_{t \bar{t}}$ values of up to $4 \mathrm{TeV}$, both processes bring small corrections relative to the QCD LO $t \bar{t}$ cross section, at most at the few-percent level. At a $100 \mathrm{TeV}$ collider, which is beyond the scope of this article, considering $m_{t \bar{t}} \geq 20 \mathrm{TeV}$, the $1 / m_{\text {top }}^{2} m_{t \bar{t}}^{2}$ scaling results in the 4 -top and EW processes becoming comparable to normal QCD $t \bar{t}$ production.

\footnotetext{
${ }^{21}$ To quantify this, care is needed in specifying a definition of $m_{t \bar{t}}$ when there are four tops from which to choose.
} 
We have also explicitly verified the stability of the $\Delta y_{t \bar{t}}$ distribution from NLO to NNLO using the MiNNLO event sample of ref. [55] across a range of $m^{t \bar{t}} / 2$ values. ${ }^{22}$ Relative to a fixed-order (MCFM) NLO calculation with a scale choice of $\mu=m^{t \bar{t}} / 2$ that is similar to that of the MiNNLO sample, we see a further substantial correction from the NNLO terms at large $\Delta y_{t \bar{t}}$. It would be interesting to further investigate this with a scale choice such as $H_{T}^{t \bar{t}+\text { jets }} / 2$ or $m_{T}^{J \text {,avg }}$ that tracks the transverse momenta of the tops across the full $\Delta y_{t \bar{t}}$ range.

We encourage further investigation of all the issues discussed in this appendix.

Open Access. This article is distributed under the terms of the Creative Commons Attribution License (CC-BY 4.0), which permits any use, distribution and reproduction in any medium, provided the original author(s) and source are credited.

\section{References}

[1] G. Degrassi et al., Higgs mass and vacuum stability in the Standard Model at NNLO, JHEP 08 (2012) 098 [arXiv: 1205.6497] [INSPIRE].

[2] S. Alekhin, J. Blümlein, S. Moch and R. Placakyte, Parton distribution functions, $\alpha_{s}$, and heavy-quark masses for LHC Run II, Phys. Rev. D 96 (2017) 014011 [arXiv:1701.05838] [INSPIRE].

[3] NNPDF collaboration, Parton distributions from high-precision collider data, Eur. Phys. J. $C 77$ (2017) 663 [arXiv: 1706.00428] [InSPIRE].

[4] T.-J. Hou et al., Progress in the CTEQ-TEA NNLO global QCD analysis, arXiv:1908.11394 [INSPIRE].

[5] S. Bailey, T. Cridge, L.A. Harland-Lang, A.D. Martin and R.S. Thorne, Parton distributions from LHC, HERA, Tevatron and fixed target data: MSHT20 PDFs, Eur. Phys. J. C 81 (2021) 341 [arXiv : 2012.04684] [INSPIRE].

[6] O. Amat et al., Impact of LHC top-quark pair measurements to CTEQ-TEA PDF analysis, arXiv: 1908.06441 [INSPIRE].

[7] S. Bailey and L. Harland-Lang, Differential Top Quark Pair Production at the LHC: Challenges for PDF Fits, Eur. Phys. J. C 80 (2020) 60 [arXiv:1909.10541] [INSPIRE].

[8] M. Czakon et al., An exploratory study of the impact of CMS double-differential top distributions on the gluon parton distribution function, J. Phys. G 48 (2020) 015003 [arXiv: 1912.08801] [INSPIRE].

[9] D. Barducci et al., Interpreting top-quark LHC measurements in the standard-model effective field theory, arXiv:1802.07237 [INSPIRE].

[10] N.P. Hartland et al., A Monte Carlo global analysis of the Standard Model Effective Field Theory: the top quark sector, JHEP 04 (2019) 100 [arXiv:1901.05965] [INSPIRE].

[11] I. Brivio, et al., O new physics, where art thou? A global search in the top sector, JHEP 02 (2020) 131 [arXiv: 1910.03606] [inSPIRE].

[12] S. Bißmann, C. Grunwald, G. Hiller and K. Kröninger, Top and Beauty synergies in SMEFT-fits at present and future colliders, arXiv:2012.10456 [INSPIRE].

\footnotetext{
${ }^{22}$ We are grateful to the authors for providing us with the event sample.
} 
[13] J. Ellis, M. Madigan, K. Mimasu, V. Sanz and T. You, Top, Higgs, Diboson and Electroweak Fit to the Standard Model Effective Field Theory, JHEP 04 (2021) 279 [arXiv:2012.02779] [INSPIRE].

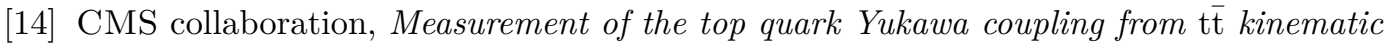
distributions in the dilepton final state in proton-proton collisions at $\sqrt{s}=13 \mathrm{TeV}$, Phys. Rev. D 102 (2020) 092013 [arXiv:2009.07123] [inSPIRE].

[15] P. Azzi et al., Report from Working Group 1: Standard Model Physics at the HL-LHC and HE-LHC, CERN Yellow Rep. Monogr. 7 (2019) 1 [arXiv:1902.04070] [INSPIRE].

[16] ATLAS collaboration, Measurements of t $\bar{t}$ differential cross-sections of highly boosted top quarks decaying to all-hadronic final states in pp collisions at $\sqrt{s}=13 \mathrm{TeV}$ using the ATLAS detector, Phys. Rev. D 98 (2018) 012003 [arXiv:1801.02052] [INSPIRE].

[17] ATLAS collaboration, Measurements of top-quark pair differential and double-differential cross-sections in the $\ell+j e t s$ channel with pp collisions at $\sqrt{s}=13 \mathrm{TeV}$ using the ATLAS detector, Eur. Phys. J. C 79 (2019) 1028 [Erratum ibid. 80 (2020) 1092] [arXiv: 1908.07305] [INSPIRE].

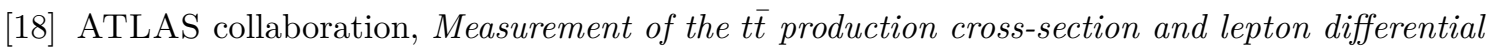

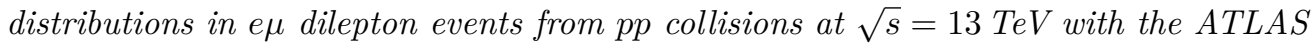
detector, Eur. Phys. J. C 80 (2020) 528 [arXiv:1910.08819] [INSPIRE].

[19] CMS collaboration, Measurement of differential t $\mathrm{t}$ production cross sections using top quarks at large transverse momenta in pp collisions at $\sqrt{s}=13 \mathrm{TeV}$, Phys. Rev. D 103 (2021) 052008 [arXiv: 2008.07860] [INSPIRE].

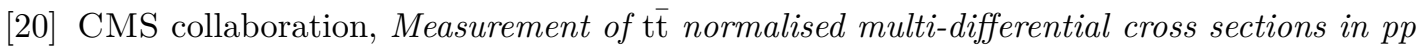
collisions at $\sqrt{s}=13 \mathrm{TeV}$, and simultaneous determination of the strong coupling strength, top quark pole mass, and parton distribution functions, Eur. Phys. J. C 80 (2020) 658 [arXiv: 1904.05237] [INSPIRE].

[21] CMS collaboration, Measurement of differential cross sections for the production of top quark pairs and of additional jets in lepton+jets events from pp collisions at $\sqrt{s}=13 \mathrm{TeV}$, Phys. Rev. D 97 (2018) 112003 [arXiv:1803.08856] [INSPIRE].

[22] G. Heinrich, Collider Physics at the Precision Frontier, arXiv:2009.00516 [INSPIRE].

[23] M. Czakon, D. Heymes and A. Mitov, Dynamical scales for multi-TeV top-pair production at the LHC, JHEP 04 (2017) 071 [arXiv: 1606. 03350] [INSPIRE].

[24] S. Catani, S. Devoto, M. Grazzini, S. Kallweit, J. Mazzitelli and H. Sargsyan, Top-quark pair hadroproduction at next-to-next-to-leading order in QCD, Phys. Rev. D 99 (2019) 051501 [arXiv: 1901.04005] [INSPIRE].

[25] ATLAS collaboration, Search for squarks and gluinos in final states with one isolated lepton, jets, and missing transverse momentum at $\sqrt{s}=13 \mathrm{TeV}$ with the ATLAS detector, arXiv:2101.01629 [INSPIRE].

[26] CMS collaboration, Running of the top quark mass from proton-proton collisions at $\sqrt{s}=13$ TeV, Phys. Lett. B 803 (2020) 135263 [arXiv:1909.09193].

[27] M.L. Mangano et al., Physics at a $100 \mathrm{TeV}$ pp Collider: Standard Model Processes, arXiv: 1607.01831 [INSPIRE].

[28] J. Butterworth et al., PDF4LHC recommendations for LHC Run II, J. Phys. G 43 (2016) 023001 [arXiv: 1510.03865] [INSPIRE]. 
[29] G.P. Salam and J. Rojo, A Higher Order Perturbative Parton Evolution Toolkit (HOPPET), Comput. Phys. Commun. 180 (2009) 120 [arXiv:0804.3755] [INSPIRE].

[30] S. Moch, J.A.M. Vermaseren and A. Vogt, The Three loop splitting functions in QCD: The Nonsinglet case, Nucl. Phys. B 688 (2004) 101 [hep-ph/0403192] [InSPIRE].

[31] A. Vogt, S. Moch and J.A.M. Vermaseren, The Three-loop splitting functions in QCD: The Singlet case, Nucl. Phys. B 691 (2004) 129 [hep-ph/0404111] [InSPIRE].

[32] M. Buza, Y. Matiounine, J. Smith, R. Migneron and W.L. van Neerven, Heavy quark coefficient functions at asymptotic values $Q^{2} \gg m^{2}$, Nucl. Phys. B $\mathbf{4 7 2}$ (1996) 611 [hep-ph/9601302] [INSPIRE].

[33] M. Buza, Y. Matiounine, J. Smith and W.L. van Neerven, Charm electroproduction viewed in the variable flavor number scheme versus fixed order perturbation theory, Eur. Phys. J. C 1 (1998) 301 [hep-ph/9612398] [INSPIRE].

[34] B.L. Combridge, J. Kripfganz and J. Ranft, Hadron Production at Large Transverse Momentum and QCD, Phys. Lett. B 70 (1977) 234 [INSPIRE].

[35] R.K. Ellis, W.J. Stirling and B.R. Webber, QCD and collider physics, Camb. Monogr. Part. Phys. Nucl. Phys. Cosmol. 8 (1996) 1.

[36] S. Catani, S. Dittmaier, M.H. Seymour and Z. Trócsányi, The Dipole formalism for next-to-leading order QCD calculations with massive partons, Nucl. Phys. B 627 (2002) 189 [hep-ph/0201036] [INSPIRE].

[37] M. Dasgupta, L. Magnea and G.P. Salam, Non-perturbative QCD effects in jets at hadron colliders, JHEP 02 (2008) 055 [arXiv:0712.3014] [INSPIRE].

[38] A. Banfi, G.P. Salam and G. Zanderighi, Accurate QCD predictions for heavy-quark jets at the Tevatron and LHC, JHEP 07 (2007) 026 [arXiv:0704.2999] [INSPIRE].

[39] M. Rubin, G.P. Salam and S. Sapeta, Giant QCD K-factors beyond NLO, JHEP 09 (2010) 084 [arXiv: 1006.2144$]$ [INSPIRE].

[40] M. Cacciari, G.P. Salam and G. Soyez, The anti- $k_{t}$ jet clustering algorithm, JHEP 04 (2008) 063 [arXiv: 0802.1189] [INSPIRE].

[41] G. Soyez, Optimal jet radius in kinematic dijet reconstruction, JHEP 07 (2010) 075 [arXiv: 1006.3634] [INSPIRE].

[42] CMS collaboration, Search for Resonances in the Dijet Mass Spectrum from $7 \mathrm{TeV} \mathrm{pp}$ Collisions at CMS, Phys. Lett. B 704 (2011) 123 [arXiv:1107.4771] [INSPIRE].

[43] J.M. Butterworth, A.R. Davison, M. Rubin and G.P. Salam, Jet substructure as a new Higgs search channel at the LHC, AIP Conf. Proc. 1078 (2009) 189 [arXiv:0809.2530] [InSPIRE].

[44] D. Krohn, J. Thaler and L.-T. Wang, Jet Trimming, JHEP 02 (2010) 084 [arXiv:0912.1342] [INSPIRE].

[45] M. Cacciari, J. Rojo, G.P. Salam and G. Soyez, Quantifying the performance of jet definitions for kinematic reconstruction at the LHC, JHEP 12 (2008) 032 [arXiv:0810.1304] [INSPIRE].

[46] S. Frixione, P. Nason and G. Ridolfi, A Positive-weight next-to-leading-order Monte Carlo for heavy flavour hadroproduction, JHEP 09 (2007) 126 [arXiv:0707.3088] [INSPIRE].

[47] S. Alioli, P. Nason, C. Oleari and E. Re, A general framework for implementing NLO calculations in shower Monte Carlo programs: the POWHEG BOX, JHEP 06 (2010) 043 [arXiv: 1002 .2581] [INSPIRE]. 
[48] A. Buckley et al., LHAPDF6: parton density access in the LHC precision era, Eur. Phys. J. C 75 (2015) 132 [arXiv: 1412.7420] [INSPIRE].

[49] T. Sjöstrand et al., An introduction to PYTHIA 8.2, Comput. Phys. Commun. 191 (2015) 159 [arXiv: 1410.3012] [inSPIRE].

[50] P. Skands, S. Carrazza and J. Rojo, Tuning PYTHIA 8.1: the Monash 2013 Tune, Eur. Phys. J. C 74 (2014) 3024 [arXiv: 1404.5630] [InSPIRE].

[51] M. Cacciari, G.P. Salam and G. Soyez, FastJet User Manual, Eur. Phys. J. C 72 (2012) 1896 [arXiv: 1111.6097] [INSPIRE].

[52] M. Czakon, D. Heymes and A. Mitov, High-precision differential predictions for top-quark pairs at the LHC, Phys. Rev. Lett. 116 (2016) 082003 [arXiv: 1511.00549] [INSPIRE].

[53] M. Czakon, P. Fiedler, D. Heymes and A. Mitov, NNLO QCD predictions for fully-differential top-quark pair production at the Tevatron, JHEP 05 (2016) 034 [arXiv: 1601.05375] [INSPIRE].

[54] S. Catani, S. Devoto, M. Grazzini, S. Kallweit and J. Mazzitelli, Top-quark pair production at the LHC: Fully differential QCD predictions at NNLO, JHEP 07 (2019) 100 [arXiv: 1906.06535] [INSPIRE].

[55] J. Mazzitelli, P.F. Monni, P. Nason, E. Re, M. Wiesemann and G. Zanderighi, Next-to-next-to-leading order event generation for top-quark pair production, arXiv: 2012.14267 [INSPIRE].

[56] S. Alioli, S.-O. Moch and P. Uwer, Hadronic top-quark pair-production with one jet and parton showering, JHEP 01 (2012) 137 [arXiv:1110.5251] [INSPIRE].

[57] A.J. Larkoski, I. Moult and B. Nachman, Jet Substructure at the Large Hadron Collider: A Review of Recent Advances in Theory and Machine Learning, Phys. Rept. 841 (2020) 1 [arXiv: 1709.04464] [INSPIRE].

[58] R. Kogler et al., Jet Substructure at the Large Hadron Collider: Experimental Review, Rev. Mod. Phys. 91 (2019) 045003 [arXiv: 1803.06991] [INSPIRE].

[59] S. Marzani, G. Soyez and M. Spannowsky, Looking inside jets: an introduction to jet substructure and boosted-object phenomenology, Lect. Notes Phys. 958 (2019) 1 [arXiv: 1901.10342] [INSPIRE].

[60] S. Chatterjee, R. Godbole and T.S. Roy, Jets with electrons from boosted top quarks, JHEP 01 (2020) 170 [arXiv: 1909.11041] [inSPIRE].

[61] S. Catani, Y.L. Dokshitzer, M.H. Seymour and B.R. Webber, Longitudinally invariant $K_{t}$ clustering algorithms for hadron hadron collisions, Nucl. Phys. B 406 (1993) 187 [INSPIRE].

[62] M.H. Seymour, Searches for new particles using cone and cluster jet algorithms: A Comparative study, Z. Phys. C 62 (1994) 127 [INSPIRE].

[63] L. Apolinário, J.G. Milhano, G.P. Salam and C.A. Salgado, Probing the time structure of the quark-gluon plasma with top quarks, Phys. Rev. Lett. 120 (2018) 232301 [arXiv:1711.03105] [INSPIRE].

[64] D.E. Kaplan, K. Rehermann, M.D. Schwartz and B. Tweedie, Top Tagging: A Method for Identifying Boosted Hadronically Decaying Top Quarks, Phys. Rev. Lett. 101 (2008) 142001 [arXiv:0806.0848] [INSPIRE]. 
[65] D. Krohn, J. Thaler and L.-T. Wang, Jets with Variable R, JHEP 06 (2009) 059 [arXiv:0903.0392] [INSPIRE].

[66] M. Dasgupta, A. Fregoso, S. Marzani and G.P. Salam, Towards an understanding of jet substructure, JHEP 09 (2013) 029 [arXiv: 1307.0007] [INSPIRE].

[67] A.J. Larkoski, S. Marzani, G. Soyez and J. Thaler, Soft Drop, JHEP 05 (2014) 146 [arXiv: 1402.2657] [INSPIRE].

[68] A. Banfi, G.P. Salam and G. Zanderighi, Infrared safe definition of jet flavor, Eur. Phys. J. C 47 (2006) 113 [hep-ph/0601139] [INSPIRE].

[69] E.A. Kuraev, L.N. Lipatov and V.S. Fadin, The Pomeranchuk Singularity in Nonabelian Gauge Theories, Sov. Phys. JETP 45 (1977) 199 [InSPIRE].

[70] I.I. Balitsky and L.N. Lipatov, The Pomeranchuk Singularity in Quantum Chromodynamics, Sov. J. Nucl. Phys. 28 (1978) 822 [INSPIRE].

[71] G. Marchesini and A.H. Mueller, BFKL dynamics in jet evolution, Phys. Lett. B 575 (2003) 37 [hep-ph/0308284] [INSPIRE].

[72] CMS collaboration, Object definitions for top quark analyses at the particle level, CMS-NOTE-2017-004, CERN-CMS-NOTE-2017-004 (2017).

[73] M. Cacciari, M. Greco and P. Nason, The $p_{T}$ spectrum in heavy flavor hadroproduction, JHEP 05 (1998) 007 [hep-ph/9803400] [INSPIRE].

[74] M. Cacciari, Top production at large $p_{t}$ at $N L O+N L L$ accuracy, PoS(DIS2018) 133 [arXiv: 1809.06626] [INSPIRE].

[75] A. Sen, Asymptotic Behavior of the Fermion and Gluon Exchange Amplitudes in Massive Quantum Electrodynamics in the Regge Limit, Phys. Rev. D 27 (1983) 2997 [INSPIRE].

[76] V.S. Fadin and V.E. Sherman, Fermion Reggeization in Nonabelian Calibration Theories, Pisma Zh. Eksp. Teor. Fiz. 23 (1976) 599.

[77] V.S. Fadin and V.E. Sherman, Processes Involving Fermion Exchange in Nonabelian Gauge Theories, Zh. Eksp. Teor. Fiz. 72 (1977) 1640 [inSPIRE].

[78] A.V. Bogdan and V.S. Fadin, A Proof of the reggeized form of amplitudes with quark exchanges, Nucl. Phys. B $\mathbf{7 4 0}$ (2006) 36 [hep-ph/0601117] [InSPIRE].

[79] J. Bartels, L.N. Lipatov and K. Peters, The Pomeranchuk singularity and vector boson reggeization in electroweak theory, Nucl. Phys. B 772 (2007) 103 [hep-ph/0610303] [INSPIRE].

[80] A.H. Mueller and P. Nason, Heavy particle content in QCD jets, Nucl. Phys. B 266 (1986) 265 [INSPIRE].

[81] M.L. Mangano and P. Nason, Heavy quark multiplicities in gluon jets, Phys. Lett. B 285 (1992) 160 [INSPIRE].

[82] M.H. Seymour, Heavy quark pair multiplicity in $e^{+} e^{-}$events, Nucl. Phys. B 436 (1995) 163 [INSPIRE].

[83] M.H. Seymour, Heavy quark production in jets, Z. Phys. C 63 (1994) 99 [INSPIRE].

[84] G. Marchesini and E. Onofri, Exact solution of BFKL equation in jet-physics, JHEP 07 (2004) 031 [hep-ph/0404242] [inSPIRE]. 
[85] G. Marchesini and A.H. Mueller, The BMS equation and cc production; a comparison of the $B M S$ and BK equations, JHEP 02 (2016) 010 [arXiv:1510.08763] [INSPIRE].

[86] R. Kirschner and L.n. Lipatov, Double Logarithmic Asymptotics and Regge Singularities of Quark Amplitudes with Flavor Exchange, Nucl. Phys. B 213 (1983) 122 [INSPIRE].

[87] B.I. Ermolaev, S.I. Manaenkov and M.G. Ryskin, Nonsinglet structure functions at small $x$, Z. Phys. C 69 (1996) 259 [hep-ph/9502262] [InSPIRE].

[88] J.M. Campbell and R.K. Ellis, An Update on vector boson pair production at hadron colliders, Phys. Rev. D 60 (1999) 113006 [hep-ph/9905386] [INSPIRE].

[89] J. Blumlein and A. Vogt, On the behavior of nonsinglet structure functions at small x, Phys. Lett. B 370 (1996) 149 [hep-ph/9510410] [INSPIRE].

[90] G. Curci, W. Furmanski and R. Petronzio, Evolution of Parton Densities Beyond Leading Order: The Nonsinglet Case, Nucl. Phys. B 175 (1980) 27 [InSPIRE].

[91] S. Moch, B. Ruijl, T. Ueda, J.A.M. Vermaseren and A. Vogt, Four-Loop Non-Singlet Splitting Functions in the Planar Limit and Beyond, JHEP 10 (2017) 041 [arXiv: 1707.08315] [INSPIRE].

[92] M.L. Mangano, M. Moretti, F. Piccinini, R. Pittau and A.D. Polosa, ALPGEN, a generator for hard multiparton processes in hadronic collisions, JHEP 07 (2003) 001 [hep-ph/0206293] [INSPIRE].

[93] J. Alwall et al., The automated computation of tree-level and next-to-leading order differential cross sections, and their matching to parton shower simulations, JHEP 07 (2014) 079 [arXiv: 1405.0301] [INSPIRE]. 